\title{
Solvability for a Fractional Order Three-Point Boundary Value System at Resonance
}

\author{
Zigen Ouyang and Hongliang Liu \\ School of Mathematics and Physics, University of South China, Hengyang 421001, China \\ Correspondence should be addressed to Zigen Ouyang; zigenouyang@163.com
}

Received 31 March 2014; Revised 15 May 2014; Accepted 16 May 2014; Published 11 June 2014

Academic Editor: Chuangxia Huang

Copyright (c) 2014 Z. Ouyang and H. Liu. This is an open access article distributed under the Creative Commons Attribution License, which permits unrestricted use, distribution, and reproduction in any medium, provided the original work is properly cited.

A class of fractional order three-point boundary value system with resonance is investigated in this paper. Using some techniques of inequalities, a completely new method is incorporated. We transform the problem into an integral equation with a pair of undetermined parameters. The topological degree theory is applied to determine the particular value of the parameters so that the system has a solution.

\section{Introduction}

In this paper, we consider the following fractional differential system:

$$
\begin{gathered}
D_{0+}^{\alpha} X(t)+f(t, X(t))=0, \\
0<t<1, \quad X=\left(x_{1}(t), x_{2}(t)\right), \\
X(0)=Y(0)=0, \quad X(1)=\frac{1}{\eta^{\alpha-1}} X(\eta), \quad 0<\eta<1,
\end{gathered}
$$

where $D_{0+}^{\alpha}$ is standard Riemann-Liouville fractional derivative of order $1<\alpha \leq 2,0<\eta<1$ and $f=\left(f_{1}, f_{2}\right)$ is a nonlinear two-dimension continuous vector function.

In the last few decades, many authors have focused on the dynamics of differential equations [1-7]; most of them have investigated fractional differential equations which have been applied in many fields such as physics, mechanics, chemistry, and engineering; see [8-13]. In particular, the positive solutions of the boundary value problem have attracted many authors' attention [14-25].

Recently, the existence of solutions of three-point boundary value problem

$$
\begin{gathered}
D_{0+}^{\alpha} u(t)+f(t, u(t))=0, \quad 0<t<1, \\
u(0)=0, \quad u(1)=\beta u(\eta), \quad 0<\eta<1,
\end{gathered}
$$

where $D_{0+}^{\alpha}$ is standard Riemann-Liouville fractional derivative of order $1<\alpha \leq 2$ has been studied by many authors under the case that $\beta \eta<1$. They obtained some nice results by using some fixed point theorems; see [26-28].

In [29], Ahmad and Nieto considered the existence results for following three-point boundary value problem for a coupled system of nonlinear fractional differential equations given by

$$
\begin{aligned}
& D_{0+}^{\alpha} u(t)=f\left(t, v(t), D_{0+}^{p} v(t)\right)=0, \quad 0<t<1, \\
& D_{0+}^{\beta} v(t)=g\left(t, u(t), D_{0+}^{q} u(t)\right)=0, \quad 0<t<1, \\
& u(0)=0, \quad u(1)=\gamma u(\eta), \\
& v(0)=0, \quad v(1)=\gamma v(\eta),
\end{aligned}
$$

where $1<\alpha, \beta<2, p, q, \gamma>0,0<\eta<1, \alpha-q \geq 1, \beta-p \geq$ $1, \gamma \eta^{\alpha-1}<1, \gamma \eta^{\beta-1}<1, D_{0+}^{\alpha}$ is standard Riemann-Liouville fractional derivative and $f, g:[0,1] \times R \times R \rightarrow R$ are given continuous functions. An existence result was proved in their paper by applying the Schauder fixed point theorem.

However, few authors have investigated fractional differential boundary value problems with resonance [1,2, 30-32].

In this paper, we establish some sufficient conditions for the existence of the boundary value system (1) by using intermediate value theorems. To present the main results, 
we assume that $f(t, X)=\left(f_{1}(t, X), f_{2}(t, X)\right)$ satisfies the following.

(H) $f(t, X) \in C([0,1] \times R \times R, R \times R), X=\left(x_{1}, x_{2}\right) \in$ $R \times R$. Suppose that there exist nonnegative functions $a_{i}(t), b_{i j}(t)(i, j=1,2)$, with $b_{11}(t)>0, b_{22}(t)>0$, $b_{i j}(t)(i, j=1,2, i \neq j) \leq b_{11}(t), b_{22}(t)$ for any $t \in[0,1]$ such that

$$
\left|f_{i}\left(t, t^{\alpha-1} X\right)\right| \leq a_{i}(t)+b_{i 1}(t)\left|x_{1}\right|^{p_{i}}+b_{i 2}(t)\left|x_{2}\right|^{q_{i}}, \quad i=1,2,
$$

where $0 \leq p_{i}, q_{i} \leq 1(i=1,2), q_{1}<p_{1}$, and $p_{2}<q_{2}$. For any real numbers $a$ and $b$, the functions $f_{i}\left(t, t^{\alpha-1}(u, v)\right)(i=1,2)$ satisfy

$$
\begin{gathered}
\lim _{v \rightarrow+\infty} f_{1}\left(t, t^{\alpha-1}(u, v)\right)>-\infty, \\
\lim _{v \rightarrow-\infty} f_{1}\left(t, t^{\alpha-1}(u, v)\right)<+\infty, \\
\text { for any } u \in R, \quad t \in(0,1], \\
\lim _{v \rightarrow+\infty} f_{2}\left(t, t^{\alpha-1}(u, v)\right)>-\infty, \\
\lim _{v \rightarrow-\infty} f_{2}\left(t, t^{\alpha-1}(u, v)\right)<+\infty,
\end{gathered}
$$

for any $v \in R, \quad t \in(0,1]$.

Furthermore, assume that

$$
\begin{aligned}
& \lim _{v \rightarrow+\infty} f_{1}\left(t, t^{\alpha-1}(v, u(v))\right)=+\infty, \\
& \text { for any } u(v) \geq-|v|, \quad t \in(0,1], \\
& \lim _{v \rightarrow-\infty} f_{1}\left(t, t^{\alpha-1}(v, u(v))\right)=-\infty,
\end{aligned}
$$$$
\text { for any } u(v) \leq|v|, \quad t \in(0,1],
$$$$
\lim _{v \rightarrow+\infty} f_{2}\left(t, t^{\alpha-1}(u(v), v)\right)=+\infty \text {, }
$$$$
\text { for any } u(v) \geq-|v|, \quad t \in(0,1] \text {, }
$$$$
\lim _{v \rightarrow-\infty} f_{2}\left(t, t^{\alpha-1}(u(v), v)\right)=-\infty,
$$

for any $u(v) \leq|v|, \quad t \in(0,1]$.

We have the following results.

Theorem 1. Assume that $(H)$ holds. If

$$
\max _{1 \leq i \leq 2}\left\{\int_{0}^{1} G^{*}(s, s)\left(b_{i 1}(s)+b_{i 2}(s)\right) d s\right\}<1,
$$

where

$$
\begin{aligned}
G^{*}(s, s)= & \frac{1}{\Gamma(\alpha)\left(1-\eta^{\alpha-1}\right)} \\
& \times \begin{cases}(1-s)^{\alpha-1}-(\eta-s)^{\alpha-1}, & 0 \leq s \leq \eta, \\
(1-s)^{\alpha-1}, & \eta \leq s \leq 1,\end{cases}
\end{aligned}
$$

then (1) has at least one solution in $[0,1]$.
Also, we consider the following special case of $(H)$ as follows.

$(\widetilde{H}) f(t, X) \in C([0,1] \times R \times R, R \times R), X=\left(x_{1}, x_{2}\right) \in$ $R \times R$. Suppose that there exist nonnegative functions $a_{i}(t), b_{i j}(t)(i, j=1,2)$, with $b_{11}(t)>0, b_{22}(t)>0$, $b_{i j}(t)(i, j=1,2, i \neq j) \leq b_{11}(t), b_{22}(t)$ for any $t \in[0,1]$ such that

$\left|f_{i}\left(t, t^{\alpha-1} X\right)\right| \leq a_{i}(t)+b_{i 1}(t)\left|x_{1}\right|^{p_{i}}+b_{i 2}(t)\left|x_{2}\right|^{q_{i}}, \quad i=1,2$,

where $0 \leq p_{i}, q_{i} \leq 1(i=1,2), q_{1}<p_{1}$, and $p_{2}<q_{2}$. The functions $f_{i}\left(t, t^{\alpha-1}(u, v)\right)(i=1,2)$ satisfy

$\lim _{v \rightarrow \pm \infty}\left|f_{1}\left(t, t^{\alpha-1}(u, v)\right)\right|<\infty \quad$ for any $u \in R, t \in(0,1]$,

$\lim _{u \rightarrow \pm \infty}\left|f_{2}\left(t, t^{\alpha-1}(u, v)\right)\right|<\infty \quad$ for any $v \in R, t \in(0,1]$.

Furthermore, assume that (7) and (8) hold.

From Theorem 1, we have the following corollary.

Corollary 2. Assume that $(\widetilde{H})$ and (9) hold; then (1) has at least one solution in $[0,1]$.

\section{Some Lemmas}

In this section, we first introduce some definitions and preliminary facts and some lemmas which will be used in this paper.

Definition 3 (see [21]). The fractional integral of order $\alpha>0$ of a function $y:(0, \infty) \rightarrow R$ is given by

$$
I_{0+}^{\alpha} y(t)=\frac{1}{\Gamma(\alpha)} \int_{0}^{t}(t-s)^{\alpha-1} y(s) d s
$$

provided that the right integral converges.

Definition 4 (see [21]). The standard Riemann-Liouville fractional derivative of order $\alpha>0$ of a continuous function $y:(0, \infty) \rightarrow R$ is given by

$$
D_{0+}^{\alpha} y(t)=\frac{1}{\Gamma(n-\alpha)} \frac{d^{n}}{d t^{n}} \int_{0}^{t}(t-s)^{n-\alpha-1} y(s) d s,
$$

where $n=[\alpha]+1$, provided that the right integral converges.

Lemma 5 (see [21]). Assume that $u \in C(0,1) \cap L(0,1)$ with a fractional derivative of order $\alpha>0$ that belongs to $C(0,1) \cap$ $L(0,1)$.Then

$$
I_{0+}^{\alpha} D_{0+}^{\alpha} y(t)=y(t)+C_{1} t^{\alpha-1}+C_{2} t^{\alpha-2}+\cdots+C_{n} t^{\alpha-n},
$$

for some $C_{i} \in R, i=1,2, \ldots, n$, where $n$ is the smallest integer greater than or equal to $\alpha$. 
The following lemma is a fixed point theorem in a particular Banach space:

$$
\Omega=\{(x(t), y(t)) \mid x(t), y(t) \in C([0,1], R)\},
$$

equipped with the norm

$$
\|(x(t), y(t))\|=\max \left\{\max _{t \in[0,1]}|x(t)|, \max _{t \in[0,1]}|y(t)|\right\} .
$$

It is easy to show that if $X(t) \in \Omega$, then $t^{\alpha-1} X(t) \in \Omega$.

Lemma 6 (see [33]). Let $X$ be a Banach space with $C \subset X$ closed and convex. Assume that $U$ is a relatively open subset of $C$ with $0 \in U$ and $T: \bar{U} \rightarrow C$ is completely continuous. Then either

(i) Thas a fixed point in $\bar{U}$, or

(ii) there exist an $u \in \partial U$ and $\gamma \in(0,1)$ with $u=\gamma T u$.

To use this lemma to prove our main result, we need transfer (1) into an integral operator.

Lemma 7 (see [34]). Problem (1) is equivalent to the following integral equation:

$$
X(t)=\int_{0}^{1} G(t, s) f(s, X(s)) d s+X(1) t^{\alpha-1},
$$

where

$$
\begin{aligned}
& G(t, s) \\
& \left\{\begin{array}{c}
\left(t^{\alpha-1}(1-s)^{\alpha-1}-t^{\alpha-1}(\eta-s)^{\alpha-1}\right. \\
\left.-\left(1-\eta^{\alpha-1}\right)(t-s)^{\alpha-1}\right) \times\left(\Gamma(\alpha)\left(1-\eta^{\alpha-1}\right)\right)^{-1}, \\
0 \leq s \leq \min \{t, \eta\} \leq 1 ; \\
\frac{t^{\alpha-1}(1-s)^{\alpha-1}-t^{\alpha-1}(\eta-s)^{\alpha-1}}{\Gamma(\alpha)\left(1-\eta^{\alpha-1}\right)}, \\
0 \leq t \leq s \leq \eta \leq 1 ; \\
\frac{t^{\alpha-1}(1-s)^{\alpha-1}-\left(1-\eta^{\alpha-1}\right)(t-s)^{\alpha-1}}{\Gamma(\alpha)\left(1-\eta^{\alpha-1}\right)}, \\
0 \leq \eta \leq s \leq t \leq 1 ; \\
\frac{t^{\alpha-1}(1-s)^{\alpha-1}}{\Gamma(\alpha)\left(1-\eta^{\alpha-1}\right)}, \quad 0 \leq \max \{t, \eta\} \leq s \leq 1 .
\end{array}\right.
\end{aligned}
$$

Lemma 8 (see [34]). For any $(t, s) \in[0,1] \times[0,1], G(t, s)$ is continuous, and $G(t, s)>0$ for any $(t, s) \in(0,1) \times(0,1)$.

Let

$$
G(t, s)=t^{\alpha-1} G^{*}(t, s),
$$

where

$$
\begin{aligned}
& G^{*}(t, s) \\
& \quad=\left\{\begin{array}{c}
\frac{(1-s)^{\alpha-1}-(\eta-s)^{\alpha-1}-\left(1-\eta^{\alpha-1}\right)(1-s / t)^{\alpha-1}}{\Gamma(\alpha)\left(1-\eta^{\alpha-1}\right)} \\
0 \leq s \leq \min \{t, \eta\} \leq 1 ; \\
\frac{(1-s)^{\alpha-1}-(\eta-s)^{\alpha-1}}{\Gamma(\alpha)\left(1-\eta^{\alpha-1}\right)}, \quad 0 \leq t \leq s \leq \eta \leq 1 ; \\
\frac{(1-s)^{\alpha-1}-\left(1-\eta^{\alpha-1}\right)(1-s / t)^{\alpha-1}}{\Gamma(\alpha)\left(1-\eta^{\alpha-1}\right)} \\
0 \leq \eta \leq s \leq t \leq 1 ; \\
\frac{(1-s)^{\alpha-1},}{\Gamma(\alpha)\left(1-\eta^{\alpha-1}\right)} \quad 0 \leq \max \{t, \eta\} \leq s \leq 1 .
\end{array}\right.
\end{aligned}
$$

Then (1) is equivalent to the following integral equation:

$$
X(t)=\int_{0}^{1} t^{\alpha-1} G^{*}(t, s) f(s, X(s)) d s+X(1) t^{\alpha-1} .
$$

The new Green's function $G^{*}(t, s)$ has the following properties.

Lemma 9 (see [34]). $G^{*}(t, s)$ is continuous for $(t, s) \in(0,1) \times$ $(0,1)$ and

$$
\begin{aligned}
\lim _{t \rightarrow 0^{+}} G^{*}(t, s) \\
\quad:=G^{*}(0, s) \\
= \begin{cases}\frac{1}{\Gamma(\alpha)\left(1-\eta^{\alpha-1}\right)} \\
\times\left\{(1-s)^{\alpha-1}-(\eta-s)^{\alpha-1}\right\}, & 0 \leq s \leq \eta ; \\
\frac{1}{\Gamma(\alpha)\left(1-\eta^{\alpha-1}\right)}(1-s)^{\alpha-1}, & \eta \leq s \leq 1 .\end{cases}
\end{aligned}
$$

Furthermore, $G^{*}(t, s)>0$ for $(t, s) \in(0,1) \times(0,1)$.

Lemma 10 (see [34]). $G^{*}(t, s)$ is nonincreasing with respect to $t \in[0,1]$ for any $s \in(0,1)$. In particular, for any $s \in[0,1]$, $\partial G^{*}(t, s) / \partial t \leq 0$, and $\partial G^{*}(t, s) / \partial t=0$ for $t \in[0, s]$. That is, $G^{*}(1, s) \leq G^{*}(t, s) \leq G^{*}(s, s)$, where

$$
\begin{aligned}
G^{*}(1, s)= & \frac{1}{\Gamma(\alpha)\left(1-\eta^{\alpha-1}\right)} \\
& \times \begin{cases}\eta^{\alpha-1}(1-s)^{\alpha-1}-(\eta-s)^{\alpha-1}, & 0 \leq s \leq \eta ; \\
\eta^{\alpha-1}(1-s)^{\alpha-1}, & \eta \leq s \leq 1,\end{cases}
\end{aligned}
$$

$$
\begin{aligned}
G^{*}(s, s)= & \frac{1}{\Gamma(\alpha)\left(1-\eta^{\alpha-1}\right)} \\
& \times \begin{cases}(1-s)^{\alpha-1}-(\eta-s)^{\alpha-1}, & 0 \leq s \leq \eta ; \\
(1-s)^{\alpha-1}, & \eta \leq s \leq 1 .\end{cases}
\end{aligned}
$$


Let

$$
X(t)=t^{\alpha-1} Y(t)
$$

Then $X(1)=Y(1)$, and (23) gives

$$
Y(t)=\int_{0}^{1} G^{*}(t, s) f\left(s, s^{\alpha-1} Y(s)\right) d s+Y(1) .
$$

Let

$$
W(t)=Y(t)-Y(1) .
$$

Then $Y(t)=W(t)+Y(1)$, and $W(1)=Y(1)-Y(1)=0$. From (27), (28) can be rewritten as

$$
W(t)=\int_{0}^{1} G^{*}(t, s) f\left(s, s^{\alpha-1}(W(s)+Y(1))\right) d s
$$

with $W(1)=0$. Now the integral equation (27) is equivalent to (29). It can be seen from (29) that the solution $W(t)$ of (29) is dependent on the value $Y(1)$. Now, instead of (29), we replace $Y(1)$ with a real vector $\kappa=(\mu, \nu)$ and consider

$$
W(t)=\int_{0}^{1} G^{*}(t, s) f\left(s, s^{\alpha-1}(W(s)+\kappa)\right) d s .
$$

For any $\kappa=(\mu, \nu)$, let

$$
K=\left\{W(t)=\left(w_{1}(t), w_{2}(t)\right) \in \Omega\right\},
$$

equipped with the norm $\|W(t)\|=\max \left\{\max _{t \in[0,1]} w_{1}(t)\right.$, $\left.\max _{t \in[0,1]} w_{2}(t)\right\}$. Define an operator $T$ in $K$ as follows:

$$
T W(t)=\int_{0}^{1} G^{*}(t, s) f\left(s, s^{\alpha-1}(W(s)+\kappa)\right) d s .
$$

Using a similar method of Lemmas 3.5 and 3.6 in [34], we obtain that $T$ is completely continuous in $K$, and (30) has at least a solution $W(t)$ for any given real constant vector $\kappa$; the solution $W(t)$ is dependent on the given vector $\kappa$. We note the solution $W(t):=W_{\kappa}(t)$.

\section{The Proof of Theorem 1}

From Lemma 10, for any real vector $\kappa$, the integral equation (30) has at least a solution $W(t)$. Therefore, to show that problem (1) has a solution, it remains to show that there exists a $\kappa=(\mu, \nu)$, such that $W(1)=0$, or $Y(1)=\kappa=(\mu, \nu)$.

In what follows, we will use the method of topological degree to prove our main result.

Let $D$ be an open subset of the plane $R^{2}$ with the boundary $\partial D$ being a simple closed curve; $\widetilde{T}$ is a continuous mapping from $\bar{D}=D \cup \partial D$ to $R^{2}$. Let $(c, d) \in R^{2}$. Denote by $A$ a variable point on the boundary $\partial D$. As $A$ traverses the boundary, assume that its image $\widetilde{T}(A)$ traces out a closed curve that does not pass through the point $(c, d)$. As in complex analysis, we can define the winding number of this curve with respect to $(c, d)$, by measuring the total change of the argument of the vector joining $(c, d)$ and the variable point $\widetilde{T}(A)$. For two-dimensional space, this number is equivalent to the topological degree of the mapping $\widetilde{T}$ at $(c, d)$.

We introduce a proposition from [8] as follows.
Proposition 11. If the degree of a continuous mapping $\widetilde{T}$ with respect to a point $(c, d)$ is nonzero, then the equation $\widetilde{T}(\mu, v)=$ $(c, d)$ has a solution $(\mu, v) \in D$.

From Section 2, for any parameters $\mu, \nu, \kappa=(\mu, \nu)$, there exists a solution $W_{\kappa}(t)$ of (30). At the point $t=1$, we denote $w_{1}(1):=\theta, w_{2}(1):=\vartheta$. It is obvious that the parameters $\theta$, $\vartheta$ depend on the parameters $\mu, \nu$, so we define a map $\widetilde{T}$ as follows:

$$
\widetilde{T}(\mu, \nu)=(\theta, \vartheta)
$$

Therefore, if we can find a domain $D$ with its boundary as a closed curve $L$, so that its image $\widetilde{T}(L)$ contains the point $(0,0)$ in it, then it is implied by Proposition 11 that there exists a point $\kappa_{0}=\left(\mu_{0}, \nu_{0}\right)$ in $D$ such that $\widetilde{T}\left(\mu_{0}, \nu_{0}\right)=(0,0)$. Thus, the function $Y(t)=W_{\kappa_{0}}(t)+\kappa_{0}=W(t)+Y(1)$ is a solution of (29), where

$$
W_{\kappa_{0}}(t)=\int_{0}^{1} G^{*}(t, s) f\left(s, s^{\alpha-1}\left(W_{\kappa_{0}}(s)+\kappa_{0}\right)\right) d s
$$

We now proceed to find such $L$. For convenience, we take a curve $L=P Q R S$, where $P=\left(-\mu^{*},-\nu^{*}\right), Q=\left(-\mu^{*}, \nu^{*}\right)$, $R=\left(\tilde{\mu}^{*}, v^{*}\right), S=\left(\tilde{\mu}^{*},-\nu^{*}\right)$, and $P Q, Q R, R S$, and $S P$ are a part of line. The image $\widetilde{T}(P Q R S)=P^{\prime} Q^{\prime} R^{\prime} S^{\prime}$. We want to show that the point $(\theta, \vartheta)=(0,0)$ is inside the closed curve $P^{\prime} Q^{\prime} R^{\prime} S^{\prime}$ as the parameters $\mu^{*}, \tilde{\mu}^{*}$, and $\nu^{*}$ are large enough. In fact, we will prove that the line $P^{\prime} Q^{\prime}\left(R^{\prime} S^{\prime}\right)$ lies in the left (right) side of the $\vartheta$-axis, and the line $Q^{\prime} R^{\prime}\left(S^{\prime} P^{\prime}\right)$ lies above (under) the $\theta$-axis as $\mu^{*}, \tilde{\mu}^{*}$, and $\nu^{*}$ are large enough.

Let

$$
\begin{gathered}
a_{1}=\int_{0}^{1} G^{*}(s, s) a_{1}(s) d s, \quad b_{11}=\int_{0}^{1} G^{*}(s, s) b_{11}(s) d s, \\
b_{12}=\int_{0}^{1} G^{*}(s, s) b_{12}(s) d s \\
a_{2}=\int_{0}^{1} G^{*}(s, s) a_{2}(s) d s, \quad b_{21}=\int_{0}^{1} G^{*}(s, s) b_{21}(s) d s \\
b_{22}=\int_{0}^{1} G^{*}(s, s) b_{22}(s) d s .
\end{gathered}
$$

From (9) and (H), $a_{1}, a_{2} \geq 0$ and $0<b_{11}, b_{12}, b_{21}, b_{22}<1$, and $b_{12}<b_{11}, b_{21}<b_{22}$, we may take $\mu^{*}, \tilde{\mu}^{*}$, and $\nu^{*}$ large enough satisfying

$$
\begin{aligned}
& \mu^{*}=\frac{b_{12}}{b_{11}} v^{*}, \\
& \tilde{\mu}^{*}=\frac{b_{22}}{b_{21}} v^{*} .
\end{aligned}
$$


Then, the points $P, Q, R$, and $S$ can be expressed as follows:

$$
\begin{aligned}
& P=\left(-\frac{b_{12}}{b_{11}} v^{*},-v^{*}\right), \\
& Q=\left(-\frac{b_{12}}{b_{11}} v^{*}, v^{*}\right), \\
& R=\left(\frac{b_{22}}{b_{21}} v^{*}, v^{*}\right), \\
& S=\left(\frac{b_{22}}{b_{21}} v^{*},-\nu^{*}\right) .
\end{aligned}
$$

Now the proof of Theorem 1 is reduced as the following lemmas.

Lemma 12. Suppose that $(H)$ and (9) hold. Then, for $v^{*}$ large enough, $P^{\prime}$ lies in the third quadrant.

Proof. From (30), we have

$$
W_{\kappa}(1):=W(1)=\int_{0}^{1} G^{*}(1, s) f\left(s, s^{\alpha-1}(W(s)+\kappa)\right) d s .
$$

By the definition of $\theta, \vartheta$ in (33), we may rewrite (39) as follows:

$$
\begin{aligned}
& \theta_{\kappa}=w_{1}(1)=\int_{0}^{1} G^{*}(1, s) f_{1}\left(s, s^{\alpha-1}(W(s)+\kappa)\right) d s, \\
& \vartheta_{\kappa}=w_{2}(1)=\int_{0}^{1} G^{*}(1, s) f_{2}\left(s, s^{\alpha-1}(W(s)+\kappa)\right) d s .
\end{aligned}
$$
have

$$
\begin{aligned}
& \theta_{\kappa^{*}}=w_{1}(1)=\int_{0}^{1} G^{*}(1, s) f_{1}\left(s, s^{\alpha-1}\left(W(s)+\kappa^{*}\right)\right) d s, \\
& \vartheta_{\kappa^{*}}=w_{2}(1)=\int_{0}^{1} G^{*}(1, s) f_{2}\left(s, s^{\alpha-1}\left(W(s)+\kappa^{*}\right)\right) d s .
\end{aligned}
$$

Now we will show that $\theta_{\kappa^{*}}, \vartheta_{\kappa^{*}} \rightarrow-\infty$ as $\nu^{*} \rightarrow \infty$. We only show that $\lim _{\nu^{*} \rightarrow \infty} \theta_{\kappa^{*}}=-\infty$ and the proof of $\lim _{\nu^{*} \rightarrow \infty} \vartheta_{\kappa^{*}}=$ $-\infty$ is similar. Assume on the contrary that $\varlimsup_{v^{*} \rightarrow \infty} \theta_{\kappa^{*}}=$ $l>-\infty$. Thus, there exists a sequence $\left\{\kappa_{n}\right\}=\left\{\left(\mu_{n}, v_{n}\right)\right\}, \mu_{n}=$ $\left(b_{12} / b_{11}\right) \nu_{n}<0$ such that $\lim _{v_{n} \rightarrow-\infty} \theta_{n}=l>-\infty$.

Recall that

$$
\begin{aligned}
& w_{1 \kappa_{n}}(t)=\int_{0}^{1} G^{*}(t, s) f_{1}\left(s, s^{\alpha-1}\left(W_{\kappa_{n}}(s)+\kappa_{n}\right)\right) d s, \\
& w_{2 \kappa_{n}}(t)=\int_{0}^{1} G^{*}(t, s) f_{2}\left(s, s^{\alpha-1}\left(W_{\kappa_{n}}(s)+\kappa_{n}\right)\right) d s .
\end{aligned}
$$

Now we claim that it is impossible to have

$$
f_{1}\left(t, t^{\alpha-1}\left(W_{\kappa_{n}}(t)+\kappa_{n}\right)\right) \leq 0, \quad \forall t \in[0,1]
$$

as $-v_{n}$ is sufficiently large. Indeed, assume that (43) is true. Then, by the first equation of (42), we have

$$
w_{1 \kappa_{n}}(t) \leq 0
$$

for all $t \in[0,1]$. Therefore, we obtain

$$
\lim _{\mu_{n} \rightarrow-\infty}\left(w_{1 \kappa_{n}}(t)+\mu_{n}\right)=\lim _{v_{n} \rightarrow-\infty}\left(w_{1 \kappa_{n}}(t)+\frac{b_{12}}{b_{11}} v_{n}\right)=-\infty
$$

for $t \in[0,1]$. We define some sets as follows:

$$
\begin{aligned}
& A_{n}=\left\{t \in[0,1]:\left|w_{1 \kappa_{n}}(t)+\frac{b_{12}}{b_{11}} v_{n}\right| \geq w_{2 \kappa_{n}}(t)+v_{n}\right\}, \\
& B_{n}=\left\{t \in[0,1]:\left|w_{1 \kappa_{n}}(t)+\frac{b_{12}}{b_{11}} v_{n}\right|<w_{2 \kappa_{n}}(t)+v_{n}\right\}, \\
& C_{n}=\left\{t \in[0,1]: w_{2 \kappa_{n}}(t)+v_{n} \geq 0\right\} .
\end{aligned}
$$

We have assumed in (7) that

$$
\begin{aligned}
& \lim _{v \rightarrow-\infty} f_{1}\left(t, t^{\alpha-1}(v, u(v))\right)=-\infty \\
& \text { for any } u(v) \leq|v|, \quad t \in(0,1] .
\end{aligned}
$$

It is easy to show from (42), $(H)$, and our assumption that the set $B_{n}$ is not empty, and $B_{n} \subset C_{n}$. We have the following:

$$
\begin{aligned}
\lim _{v_{n} \rightarrow-\infty}\left\|w_{2 \kappa_{n}}(t)\right\|_{B_{n}} & =\lim _{\nu_{n} \rightarrow-\infty}\left\|w_{2 \kappa_{n}}(t)\right\|_{C_{n}} \\
& =\lim _{v_{n} \rightarrow-\infty} \max _{t \in B_{n}} w_{2 \kappa_{n}}(t)=+\infty .
\end{aligned}
$$

Using conditions (6) and (8), we have from (45) that there exists a constant $l$ such that

$$
f_{2}\left(t, t^{\alpha-1}\left(w_{1 \kappa_{n}}(t)+\frac{b_{12}}{b_{11}} v_{n}, w_{2 \kappa_{n}}(t)+v_{n}\right)\right)<l
$$


for $t \in[0,1] \backslash C_{n}$ and any $n$ large enough. From the second formula of (42), (45)-(49), one gets

$$
\begin{aligned}
& w_{2 \kappa_{n}}(t) \leq\left(\int_{[0,1] \backslash C_{n}}+\int_{C_{n} \cap A_{n}}+\int_{C_{n} \cap B_{n}}\right) G^{*}(s, s) \\
& \times f_{2}\left(s, s^{\alpha-1}\left(w_{1 \kappa_{n}}(s)+\frac{b_{12}}{b_{11}} v_{n}, w_{2 \kappa_{n}}(s)+v_{n}\right)\right) d s \\
& \leq l \int_{[0,1] \backslash C_{n}} G^{*}(s, s) d s \\
& +\int_{C_{n} \cap B_{n}} G^{*}(s, s) \\
& \times f_{2}\left(s, s^{\alpha-1}\left(w_{1 \kappa_{n}}(s)+\frac{b_{12}}{b_{11}} v_{n},\right.\right. \\
& \left.\left.w_{2 \kappa_{n}}(s)\left(+v_{n}\right)\right)\right) d s \\
& =l \int_{[0,1] \backslash C_{n}} G^{*}(s, s) d s \\
& +\int_{B_{n}} G^{*}(s, s) \\
& \times f_{2}\left(s, s^{\alpha-1}\left(w_{1 \kappa_{n}}(s)+\frac{b_{12}}{b_{11}} v_{n}\right.\right. \\
& \left.\left.w_{2 \kappa_{n}}(s)+v_{n}\right)\right) d s \\
& \leq l \int_{[0,1] \backslash C_{n}} G^{*}(s, s) d s+\int_{0}^{1} G^{*}(s, s) a_{2}(s) d s \\
& +\left\|w_{1 \kappa_{n}}(t)+\frac{b_{21}}{b_{11}} v_{n}\right\|_{B_{n}}^{p_{2}} \int_{0}^{1} G^{*}(s, s) b_{21}(s) d s \\
& +\left\|w_{2 \kappa_{n}}(t)+v_{n}\right\|_{B_{n}}^{q_{2}} \int_{0}^{1} G^{*}(s, s) b_{22}(s) d s \\
& \leq l \int_{[0,1] \backslash C_{n}} G^{*}(s, s) d s+a_{2}+\left(b_{21}+b_{22}\right)\left\|w_{2 \kappa_{n}}(t)\right\|_{C_{n}},
\end{aligned}
$$

which implies that

$$
\left\|w_{2 \kappa_{n}}(t)\right\|_{C_{n}} \leq \frac{l \int_{[0,1] \backslash C_{n}} G^{*}(s, s) d s+a_{2}}{1-b_{21}-b_{22}}<\infty .
$$

It contradicts (48).

Thus, for any $-\nu_{n}$ large enough, there exists some $t \in$ $(0,1]$, such that

$$
f_{1}\left(t, t^{\alpha-1}\left(W_{\kappa_{n}}(t)+\kappa_{n}\right)\right)>0 .
$$

Now we define

$$
\begin{aligned}
& I_{n}=\left\{t \in[0,1]: f_{1}\left(t, t^{\alpha-1}\left(W_{\kappa_{n}}(t)+\kappa_{n}\right)\right)>0\right\}, \\
& I_{n}^{\prime}=\left\{t \in[0,1]: f_{2}\left(t, t^{\alpha-1}\left(W_{\kappa_{n}}(t)+\kappa_{n}\right)\right)>0\right\} .
\end{aligned}
$$

Then, $I_{n}$ is not empty.
We can further divide the set $I_{n}$ into two sets $\widetilde{I}_{n}$ and $\widehat{I}_{n}$, and divide the set $I_{n}^{\prime}$ into two sets $\widetilde{I_{n}^{\prime}}$ and $\widehat{I_{n}^{\prime}}$ as follows:

$$
\begin{aligned}
& \widetilde{I}_{n}=\left\{t \in I_{n} \mid w_{1 \kappa_{n}}(t)+\frac{b_{12}}{b_{11}} v_{n} \leq 0\right\}, \\
& \widehat{I_{n}}=\left\{t \in I_{n} \mid w_{1 \kappa_{n}}(t)+\frac{b_{12}}{b_{11}} v_{n}>0\right\}, \\
& \widetilde{I_{n}^{\prime}}=\left\{t \in I_{n}^{\prime} \mid w_{2 \kappa_{n}}(t)+v_{n} \leq 0\right\}, \\
& \widehat{I_{n}^{\prime}}=\left\{t \in I_{n}^{\prime} \mid w_{2 \kappa_{n}}(t)+v_{n}>0\right\} .
\end{aligned}
$$

It is easy to know that $\widetilde{I}_{n} \cap \widehat{I}_{n}=\phi, \widetilde{I_{n}^{\prime}} \cap \widehat{I_{n}^{\prime}}=\phi$ and $I_{n}=\widetilde{I}_{n} \cup \widehat{I}_{n}$, $I_{n}^{\prime}=\widetilde{I_{n}^{\prime}} \cup \widehat{I_{n}^{\prime}}$.

We claim that the set $\widehat{I}_{n}$ is not empty for $-v_{n}$ large enough. Otherwise, the function $f_{1}\left(t, t^{\alpha-1}\left(W_{\kappa_{n}}(t)+\kappa_{n}\right)\right)$ is bounded from above. In fact, assume that $f_{1}\left(t, t^{\alpha-1}\left(W_{\kappa_{n}}(t)+\kappa_{n}\right)\right)$ is unbounded from above for $-v_{n}$ large enough; then we have from $(H)$ that there exist a sequence $\left\{t_{i}\right\}$ and a subsequence $\left\{v_{n_{i}}\right\}$ of $\left\{v_{n}\right\}$ such that

$$
\begin{gathered}
\lim _{v_{n_{i}} \rightarrow-\infty} w_{2 \kappa_{n_{i}}}\left(t_{i}\right)=\infty, \\
\lim _{v_{n_{i}} \rightarrow-\infty}\left|w_{1 \kappa_{n_{i}}}\left(t_{i}\right)+\frac{b_{12}}{b_{11}} v_{n_{i}}\right| \\
\leq \lim _{v_{n_{i}} \rightarrow-\infty}\left(w_{2 \kappa_{n_{i}}}\left(t_{i}\right)+v_{n_{i}}\right) \\
=+\infty .
\end{gathered}
$$

Using a similar method of (51), we can derive a contradiction. Therefore, $f_{1}\left(t, t^{\alpha-1}\left(W_{\kappa_{n}}(t)+\kappa_{n}\right)\right)$ is bounded from above. From (42), $w_{1 \kappa_{n}}(t)$ is bounded from above, which implies that $w_{1 \kappa_{n}}(t)+\left(b_{12} / b_{11}\right) \nu_{n} \rightarrow-\infty$ as $\nu_{n} \rightarrow-\infty$. If $B_{n}=\phi$ (where $B_{n}$ is defined in (46)), then $\lim _{v_{n} \rightarrow-\infty} \theta_{n}=-\infty$, which contradicts our assumption. Thus, $B_{n} \neq \phi$. Using a similar method of getting (51) also gives a contradiction. Therefore, $\widehat{I}_{n}$ is not empty.

Similarly as getting (51) again, we conclude that the function $f_{i}\left(t, t^{\alpha-1} X\right)$ is bounded above by a constant for $t \epsilon$ $[0,1]$ and $x_{i} \in(-\infty, 0](i=1,2)$. From the condition $(H)$, if $w_{1 \kappa_{n}}(t)+v_{n}>0\left(\right.$ or $\left.w_{2 \kappa_{n}}(t)+\mu_{n}>0\right)$ and $f_{2}\left(t, t^{\alpha-1}\left(W_{\kappa_{n}}(t)+\right.\right.$ $\left.\left.\kappa_{n}\right)\right)<0\left(\right.$ or $\left.f_{1}\left(t, t^{\alpha-1}\left(W_{\kappa_{n}}(t)+\kappa_{n}\right)\right)<0\right)$, then $w_{2 \kappa_{n}}(t)+v_{n}$ (or $\left.w_{1 \kappa_{n}}(t)+\mu_{n}\right)$ is also bounded from above by a constant for $t \in[0,1]$. Therefore, from the definition of $\widetilde{I}_{n}, \widetilde{I_{n}^{\prime}}$, there exists a constant $M>1$, independent of $t$ and $\nu_{n}$ such that

$$
\begin{aligned}
& f_{1}\left(t, t^{\alpha-1}\left(W_{\kappa_{n}}(t)+\kappa_{n}\right)\right) \leq M, \quad \text { for } t \in \widetilde{I}_{n}, \\
& f_{2}\left(t, t^{\alpha-1}\left(W_{\kappa_{n}}(t)+\kappa_{n}\right)\right) \leq M, \quad \text { for } t \in \widetilde{I_{n}^{\prime}}, \\
& w_{2 \kappa_{n}}(t)+v_{n} \leq M \\
& \text { for } f_{2}\left(t, t^{\alpha-1}\left(W_{\kappa_{n}}(t)+\kappa_{n}\right)\right)<0, \quad t \in \widehat{I_{n}},
\end{aligned}
$$


$w_{1 \kappa_{n}}(t)+\frac{b_{12}}{b_{11}} v_{n} \leq M$

for $f_{1}\left(t, t^{\alpha-1}\left(W_{\kappa_{n}}(t)+\kappa_{n}\right)\right)<0, \quad t \in \widehat{I_{n}^{\prime}}$.

Let

$$
\bar{M}_{1}\left(\kappa_{n}\right)=\max _{t \in I_{n}} w_{1 \kappa_{n}}(t) .
$$

From the definitions of $\widetilde{I}_{n}$ and $\widehat{I_{n}}$, we have

$$
\bar{M}_{1}\left(\kappa_{n}\right)=\max _{t \in \widehat{I}_{n}} w_{1 \kappa_{n}}(t)=\left\|w_{1 \kappa_{n}}(t)\right\|_{I_{n}} \cdot
$$

Since $\widehat{I_{n}}$ is not empty, it follows that $\bar{M}_{1}\left(\kappa_{n}\right) \rightarrow \infty$ as $v_{n} \rightarrow-\infty$. Recall from (9) and (35) that $b_{i j}<1(i, j=1,2)$. Therefore, we can choose $v_{n_{1}}>0$ large enough so that

$$
\bar{M}_{1}\left(\kappa_{n}\right)>\max \left\{1, P_{1}, P_{2}\right\}
$$

for $v_{n}<-v_{n_{1}}$, where

$$
\begin{gathered}
P_{1}=\frac{M\left(\int_{0}^{1} G^{*}(s, s) d s+b_{12}\right)+a_{1}+b_{11}}{1-b_{11}}, \\
P_{2}=\left(M \int_{0}^{1} G^{*}(s, s) d s\left(1-b_{22}+b_{12}\right)\right. \\
\quad+b_{12}\left(1-b_{22}+b_{21}\right)+\left(a_{2}+b_{22}\right) b_{12} \\
\left.\quad+\left(a_{1}+b_{11}\right)\left(1-b_{22}\right)\right) \\
\quad \times\left(\left(1-b_{11}\right)\left(1-b_{22}\right)-b_{12} b_{21}\right)^{-1} .
\end{gathered}
$$

Now, for later use, for any integral in a domain $A$

$$
\int_{A} G^{*}(s, s) b_{i j}(s) g(s) d s, \quad \text { for } g(s)>0, i, j=1,2 \text {, }
$$

we define a subset $(A)_{1}$ as

$$
(A)_{1}=\{t \in A \mid g(t) \geq 1\} \text {. }
$$

Thus, the integral in (62) can be rewritten as

$$
\begin{aligned}
\int_{A} G^{*}(s, s) b_{i j}(s) g(s) d s= & \int_{(A)_{1}} G^{*}(s, s) b_{i j}(s) g(s) d s \\
& +\int_{A \backslash(A)_{1}} G^{*}(s, s) b_{i j}(s) g(s) d s .
\end{aligned}
$$

From $(H),(42)$, and the definitions of $\widetilde{I}_{n}, \widehat{I}_{n}$ and $\widetilde{I_{n}^{\prime}}, \widehat{I_{n}^{\prime}}$, for $v_{n}<-v_{n_{1}}$, we have

$$
\begin{aligned}
& w_{1 \kappa_{n}}(t)=\int_{0}^{1} G^{*}(t, s) f_{1}\left(s, s^{\alpha-1}\left(W_{\kappa_{n}}(s)+\kappa_{n}\right)\right) d s \\
& \leq \int_{I_{n}} G^{*}(t, s) f_{1}\left(s, s^{\alpha-1}\left(W_{\kappa_{n}}(s)+\kappa_{n}\right)\right) d s \\
& \leq \int_{\widetilde{I}_{n}} G^{*}(s, s) f_{1}\left(s, s^{\alpha-1}\left(W_{\kappa_{n}}(s)+\kappa_{n}\right)\right) d s \\
& +\int_{\widehat{I_{n}}} G^{*}(s, s) a_{1}(s) d s \\
& +\int_{\widehat{I}_{n}} G^{*}(s, s)\left(b_{11}(s)\left|w_{1 \kappa_{n}}(s)+\frac{b_{12}}{b_{11}} v_{n}\right|^{p_{1}}\right. \\
& \left.+b_{12}(s)\left|w_{2 \kappa_{n}}(s)+v_{n}\right|^{q_{1}}\right) d s \\
& \leq \int_{\widetilde{I}_{n}} G^{*}(s, s) f_{1}\left(s, s^{\alpha-1}\left(W_{\kappa_{n}}(s)+\kappa_{n}\right)\right) d s \\
& +\int_{\widehat{I}_{n}} G^{*}(s, s) a_{1}(s) d s \\
& +\int_{\widehat{I}_{n} \backslash\left(\widehat{I}_{n}\right)_{1}} G^{*}(s, s) b_{11}(s)\left|w_{1 \kappa_{n}}(s)+\frac{b_{12}}{b_{11}} v_{n}\right|^{p_{1}} d s \\
& +\int_{\left(\widehat{I}_{n}\right)_{1}} G^{*}(s, s) b_{11}(s)\left|w_{1 \kappa_{n}}(s)+\frac{b_{12}}{b_{11}} v_{n}\right|^{p_{1}} d s \\
& +\int_{\left(\widehat{I}_{n} \cap I_{n}^{\prime}\right)_{1}} G^{*}(s, s) b_{12}(s)\left|w_{2 \kappa_{n}}(s)+v_{n}\right|^{q_{1}} d s \\
& +\int_{\left(\widehat{I_{n}} \cap\left([0,1] \backslash I_{n}^{\prime}\right)\right) \cup\left(\left(\widehat{I}_{n} \cap I_{n}^{\prime}\right) \backslash\left(\widehat{I}_{n} \cap I_{n}^{\prime}\right)_{1}\right)} G^{*}(s, s) b_{12}(s) \\
& \times\left|w_{2 \kappa_{n}}(s)+v_{n}\right|^{q_{1}} d s,
\end{aligned}
$$

which yields from (56) and the definition in (63) that

$$
\begin{aligned}
& w_{1 \kappa_{n}}(t) \leq \int_{\widetilde{I}_{n}} G^{*}(s, s) f_{1}\left(s, s^{\alpha-1}\left(W_{\kappa_{n}}(s)+\kappa_{n}\right)\right) d s \\
& +\int_{\widehat{I_{n}}} G^{*}(s, s) a_{1}(s) d s+\int_{\widehat{I_{n}} \backslash\left(\widehat{I_{n}}\right)_{1}} G^{*}(s, s) b_{11}(s) d s \\
& +\int_{\left(\widehat{I}_{n}\right)_{1}} G^{*}(s, s) b_{11}(s)\left|w_{1 \kappa_{n}}(s)+\frac{b_{12}}{b_{11}} v_{n}\right| d s \\
& +\int_{\left(\widehat{I_{n}} \cap I_{n}^{\prime}\right)_{1}} G^{*}(s, s) b_{12}(s)\left|w_{2 \kappa_{n}}(s)+v_{n}\right| d s \\
& +M \int_{\left(\widehat{I_{n}} \cap\left([0,1] \backslash I_{n}^{\prime}\right)\right) \cup\left(\left(\widehat{I}_{n} \cap I_{n}^{\prime}\right) \backslash\left(\widehat{I}_{n} \cap I_{n}^{\prime}\right)_{1}\right)} G^{*}(s, s) b_{12}(s) d s .
\end{aligned}
$$


Further, one gets from (56) that

$$
\begin{aligned}
w_{1 \kappa_{n}}(t) \leq & \int_{0}^{1} G^{*}(s, s) M d s+\int_{0}^{1} G^{*}(s, s) a_{1}(s) d s \\
& +\int_{0}^{1} G^{*}(s, s) b_{11}(s) d s+M \int_{0}^{1} G^{*}(s, s) b_{12}(s) d s \\
& +\int_{0}^{1} G^{*}(s, s) b_{11}(s) d s\left(\bar{M}_{1}\left(\kappa_{n}\right)-\frac{b_{12}}{b_{11}}\left\|v_{n}\right\|\right) \\
& +\int_{0}^{1} G^{*}(s, s) b_{12}(s) d s\left(\left\|w_{2 \kappa_{n}}(t)\right\|_{\widehat{I}_{n} \cap I_{n}^{\prime}}+\left\|v_{n}\right\|\right) \\
= & M\left(\int_{0}^{1} G^{*}(s, s) d s+b_{12}\right)+a_{1}+b_{11}+b_{11} \bar{M}_{1}\left(\kappa_{n}\right) \\
& +b_{12}\left\|w_{2 \kappa_{n}}(t)\right\|_{\widehat{I}_{n} \cap I_{n}^{\prime}}
\end{aligned}
$$

which gives

$$
\begin{aligned}
\bar{M}_{1}\left(\kappa_{n}\right)< & M\left(\int_{0}^{1} G^{*}(s, s) d s+b_{12}\right)+a_{1}+b_{11}+b_{11} \bar{M}_{1}\left(\kappa_{n}\right) \\
& +b_{12}\left\|w_{2 \kappa_{n}}(t)\right\|_{\widehat{I}_{n} \cap I_{n}^{\prime}}
\end{aligned}
$$

That is,

$$
\begin{aligned}
& \bar{M}_{1}\left(\kappa_{n}\right) \\
& \quad<\frac{M\left(\int_{0}^{1} G^{*}(s, s) d s+b_{12}\right)+a_{1}+b_{11}+b_{12}\left\|w_{2 \kappa_{n}}(t)\right\|_{\widehat{I}_{n} \cap I_{n}^{\prime}}}{1-b_{11}} .
\end{aligned}
$$

If $\widehat{I_{n}} \cap I_{n}^{\prime}=\phi$, then we have from (69) that

$$
\bar{M}_{1}\left(\kappa_{n}\right)<\frac{M\left(\int_{0}^{1} G^{*}(s, s) d s+b_{12}\right)+a_{1}+b_{11}}{1-b_{11}},
$$

which contradicts (59).

If $\widehat{I_{n}} \cap I_{n}^{\prime} \neq \phi$, using a similar method of (69), we can estimate $w_{2 \kappa_{n}}(t)$ as

$$
\begin{aligned}
& \left\|w_{2 \kappa_{n}}(t)\right\|_{\widehat{I}_{n} \cap I_{n}^{\prime}} \\
& \leq\left\|w_{2 \kappa_{n}}(t)\right\|_{I_{n}^{\prime}} \\
& \quad<\frac{M\left(\int_{0}^{1} G^{*}(s, s) d s+b_{21}\right)+a_{2}+b_{22}+b_{21} \bar{M}\left(\kappa_{n}\right)}{1-b_{22}} .
\end{aligned}
$$

Substituting this into (69), we obtain

$$
\begin{aligned}
& \bar{M}\left(\kappa_{n}\right) \\
& <\left(M\left(\int_{0}^{1} G^{*}(s, s) d s\left(1-b_{22}+b_{12}\right)+b_{12}\left(1-b_{22}+b_{21}\right)\right)\right. \\
& \left.\quad+\left(a_{2}+b_{22}\right) b_{12}+\left(a_{1}+b_{11}\right)\left(1-b_{22}\right)\right) \\
& \quad \times\left(\left(1-b_{11}\right)\left(1-b_{22}\right)-b_{12} b_{21}\right)^{-1},
\end{aligned}
$$

which finally contradicts (59). Therefore, our result is proved.

Similarly, we can show that $\lim _{v^{*} \rightarrow \infty} \vartheta_{\kappa^{*}}=-\infty$. Thus, the point $\widetilde{T}\left(-\mu^{*},-v^{*}\right)$ lies in the third quadrant. The proof is completed.

Lemma 13. Suppose that $(H)$ and (9) hold. Then, for $v^{*}>0$ large enough, $Q^{\prime}$ lies in the second quadrant.

Proof. It suffices to show that $\lim _{\nu^{*} \rightarrow \infty} \vartheta^{*}=\infty$ and $\lim _{v^{*} \rightarrow \infty} \theta^{*}=-\infty$.

First, we claim that $\lim _{\nu^{*} \rightarrow \infty} \vartheta^{*}=\infty$. On the contrary, we assume that there exists a sequence $\left\{\kappa_{n}\right\}=\left\{\left(\mu_{n}, \nu_{n}\right)\right\}=$ $\left\{\left(-\left(b_{12} / b_{11}\right) v_{n}, v_{n}\right)\right\}$ such that $\lim _{v_{n} \rightarrow \infty} \vartheta_{n}=l<\infty$. By a similar method in Lemma 12, we know it is impossible to have

$$
f_{2}\left(t, t^{\alpha-1}\left(W_{\kappa_{n}}(t)+\kappa_{n}\right)\right) \geq 0, \quad \forall t \in[0,1]
$$

as $\nu_{n}$ is sufficiently large.

Now, for large $v_{n}$, we define

$$
\begin{aligned}
& J_{n}=\left\{t \in[0,1]: f_{1}\left(t, t^{\alpha-1}\left(W_{\kappa_{n}}(t)+\kappa_{n}\right)\right)<0\right\}, \\
& J_{n}^{\prime}=\left\{t \in[0,1]: f_{2}\left(t, t^{\alpha-1}\left(W_{\kappa_{n}}(t)+\kappa_{n}\right)\right)<0\right\} .
\end{aligned}
$$

Then, $J_{n}^{\prime}$ is not empty.

As in Lemma 12, we can further divide the set $J_{n}$ into two sets $\widetilde{J_{n}}$ and $\widehat{J_{n}}$ and divide the set $J_{n}^{\prime}$ into two sets $\widetilde{J_{n}^{\prime}}$ and $\widehat{J_{n}^{\prime}}$ as follows:

$$
\begin{aligned}
& \widetilde{J_{n}}=\left\{t \in J_{n} \mid w_{1 \kappa_{n}}(t)-\frac{b_{12}}{b_{11}} v_{n} \geq 0\right\}, \\
& \widehat{J_{n}}=\left\{t \in J_{n} \mid w_{1 \kappa_{n}}(t)-\frac{b_{12}}{b_{11}} v_{n}<0\right\}, \\
& \widetilde{J_{n}^{\prime}}=\left\{t \in J_{n}^{\prime} \mid w_{2 \kappa_{n}}(t)+v_{n} \geq 0\right\}, \\
& \widehat{J_{n}^{\prime}}=\left\{t \in J_{n}^{\prime} \mid w_{2 \kappa_{n}}(t)+v_{n}<0\right\} .
\end{aligned}
$$

Then $\widetilde{J_{n}} \cap \widehat{J_{n}}=\phi, \widetilde{J_{n}^{\prime}} \cap \widehat{J_{n}^{\prime}}=\phi$ and $J_{n}=\widetilde{J_{n}} \cup \widehat{J_{n}}, J_{n}^{\prime}=\widetilde{J_{n}^{\prime}} \cup \widehat{J_{n}^{\prime}}$.

Using a similar method as in the proof of Lemma 12, we can show that the set $\widehat{J_{n}^{\prime}}$ is not empty. Furthermore, the function $f_{i}\left(t, t^{\alpha-1} X\right)$ is bounded below by a constant for $t \epsilon$ $[0,1]$ and $x_{i} \in[0, \infty)(i=1,2)$. If $w_{2 \kappa_{n}}(t)+\mu_{n}<0$ (or $w_{1 \kappa_{n}}(t)+v_{n}<0$ ) and $f_{1}\left(t, t^{\alpha-1}\left(W_{\kappa_{n}}(t)+\kappa_{n}\right)\right)>0$ (or $\left.f_{2}\left(t, t^{\alpha-1}\left(W_{\kappa_{n}}(t)+\kappa_{n}\right)\right)>0\right)$, then $w_{1 \kappa_{n}}(t)+v_{n}\left(\right.$ or $\left.w_{2 \kappa_{n}}(t)+\mu_{n}\right)$ 
is also bounded below by a constant for $t \in[0,1]$. From the definition of $\widetilde{J_{n}}, \widetilde{J_{n}^{\prime}}$ and the condition $(H)$, there exists a constant $\widetilde{M}<-1$, independent of $t$ and $v_{n}$ such that

$$
\begin{aligned}
& f_{1}\left(t, t^{\alpha-1}\left(W_{\kappa_{n}}(t)+\kappa_{n}\right)\right) \geq \widetilde{M}, \quad \text { for } t \in \widetilde{J_{n}}, \\
& f_{2}\left(t, t^{\alpha-1}\left(W_{\kappa_{n}}(t)+\kappa_{n}\right)\right) \geq \widetilde{M}, \quad \text { for } t \in \widetilde{J_{n}^{\prime}}, \\
& w_{2 \kappa_{n}}(t)+v_{n} \geq \widetilde{M} \\
& \text { for } f_{2}\left(t, t^{\alpha-1}\left(W_{\kappa_{n}}(t)+\kappa_{n}\right)\right)>0, \quad t \in \widehat{J_{n}}, \\
& w_{1 \kappa_{n}}(t)-\frac{b_{12}}{b_{11}} v_{n} \geq \widetilde{M}, \\
& \text { for } f_{1}\left(t, t^{\alpha-1}\left(W_{\kappa_{n}}(t)+\kappa_{n}\right)\right)>0, \quad t \in \widehat{J_{n}^{\prime} .}
\end{aligned}
$$

Let

$$
\bar{m}_{2}\left(\kappa_{n}\right)=\min _{t \in J_{n}^{\prime}} w_{2 \kappa_{n}}(t)
$$

From the definitions of $\widetilde{J_{n}^{\prime}}$ and $\widehat{J_{n}^{\prime}}$, we have

$$
\bar{m}_{2}\left(\kappa_{n}\right)=\min _{t \in \widehat{J}_{n}^{\prime}} w_{2 \kappa_{n}}(t)=-\left\|w_{2 \kappa_{n}}(t)\right\|_{J_{n}^{\prime}},
$$

and it follows that $\bar{m}_{2}\left(\kappa_{n}\right) \rightarrow-\infty$ as $\nu_{n} \rightarrow \infty$. Therefore, we can choose $v_{n_{1}}$ large enough so that

$$
\bar{m}_{2}\left(\kappa_{n}\right)<\min \left\{-1, Q_{1}, Q_{2}\right\}
$$

for $v_{n}>v_{n_{1}}$, where

$$
\begin{aligned}
Q_{1}= & \frac{\widetilde{M} \int_{0}^{1} G^{*}(s, s) d s\left(1+b_{21}\right)-a_{2}-b_{22}}{1-b_{22}}, \\
Q_{2}= & \frac{\int_{0}^{1} G^{*}(s, s) d s\left(1-b_{11}-b_{21}\right)+b_{21}\left(1-b_{11}-b_{21}\right)}{\left(1-b_{22}\right)\left(1-b_{11}\right)-b_{21} b_{12}} \widetilde{M} \\
& -\frac{\left(a_{2}+b_{22}\right)\left(1-b_{11}\right)+\left(a_{1}+b_{11}\right) b_{21}}{\left(1-b_{22}\right)\left(1-b_{11}\right)-b_{21} b_{12}} .
\end{aligned}
$$

Notice that $b_{12}<b_{11}, b_{21}<b_{22}$. From $(H),(42)$, and the definitions of $\widetilde{J_{n}}, \widehat{J_{n}}$ and $\widetilde{J_{n}^{\prime}}, \widehat{J_{n}^{\prime}}$, for $v_{n}>v_{n_{1}}$, we have

$$
\begin{aligned}
& w_{2 \kappa_{n}}(t) \geq \int_{J_{n}^{\prime}} G^{*}(s, s) f_{2}\left(s, s^{\alpha-1}\left(W_{\kappa_{n}}(s)+\kappa_{n}\right)\right) d s \\
& \geq \int_{\widetilde{J}_{n}^{\prime}} G^{*}(s, s) f_{2}\left(s, s^{\alpha-1}\left(W_{\kappa_{n}}(s)+\kappa_{n}\right)\right) d s \\
& -\int_{\widehat{J_{n}^{\prime}}} G^{*}(s, s) a_{2}(s) d s \\
& -\int_{\widehat{J}_{n}^{\prime}} G^{*}(s, s)\left(b_{21}(s)\left|w_{1 \kappa_{n}}(s)-\frac{b_{12}}{b_{11}} v_{n}\right|^{p_{2}}\right. \\
& \left.+b_{22}(s)\left|w_{2 \kappa_{n}}(s)+v_{n}\right|^{q_{2}}\right) d s \\
& \geq \int_{\widetilde{T}_{n}^{\prime}} G^{*}(s, s) f_{2}\left(s, s^{\alpha-1}\left(W_{\kappa_{n}}(s)+\kappa_{n}\right)\right) d s \\
& -\int_{\widehat{J_{n}^{\prime}}} G^{*}(s, s) a_{2}(s) d s \\
& -\int_{\widehat{J}_{n}^{\top} \cap J_{n}} G^{*}(s, s) b_{21}(s)\left|w_{1 \kappa_{n}}(s)-\frac{b_{12}}{b_{11}} v_{n}\right|^{p_{2}} d s \\
& -\int_{\widehat{J_{n}^{\prime}} \cap\left([0,1] \backslash \backslash_{n}\right)} G^{*}(s, s) b_{21}(s)\left|w_{1 \kappa_{n}}(s)-\frac{b_{12}}{b_{11}} v_{n}\right|^{p_{2}} d s \\
& -\int_{\widehat{J_{n}^{\prime}}} G^{*}(s, s) b_{22}(s)\left|w_{2 \kappa_{n}}(s)+v_{n}\right|^{q_{2}} d s .
\end{aligned}
$$

Thus,

$$
\begin{aligned}
& w_{2 \kappa_{n}}(t) \\
& \geq \int_{{\widetilde{J_{n}^{\prime}}}^{\prime}} G^{*}(s, s) f_{2}\left(s, s^{\alpha-1}\left(W_{\kappa_{n}}(s)+\kappa_{n}\right)\right) d s \\
& -\int_{\widehat{J_{n}^{\prime}}} G^{*}(s, s) a_{2}(s) d s \\
& -\int_{\left(\widehat{J}_{n}^{\top} \cap J_{n}\right)_{1}} G^{*}(s, s) b_{21}(s)\left|w_{1 \kappa_{n}}(s)-\frac{b_{12}}{b_{11}} v_{n}\right|^{p_{2}} d s \\
& -\int_{\left.\left(\widehat{J_{n}} \cap\left([0,1] \backslash J_{n}\right)\right) \cup\left(\widehat{J_{n}^{\prime}} \cap J_{n}\right) \backslash\left(\widehat{J_{n}^{\prime}} \cap J_{n}\right)_{1}\right)} G^{*}(s, s) b_{21}(s) \\
& \times\left|w_{1 \kappa_{n}}(s)-\frac{b_{12}}{b_{11}} v_{n}\right|^{p_{2}} d s \\
& -\int_{\left(\widehat{\left.J_{n}^{\prime}\right)_{1}}\right.} G^{*}(s, s) b_{22}(s)\left|w_{2 \kappa_{n}}(s)+v_{n}\right|^{q_{2}} d s \\
& -\int_{\widehat{J_{n}^{\prime}}\left(\widehat{\left.J_{n}^{\prime}\right)_{1}}\right.} G^{*}(s, s) b_{22}(s)\left|w_{2 \kappa_{n}}(s)+v_{n}\right|^{q_{2}} d s,
\end{aligned}
$$


which follows from (76) and the definition of (63) that

$$
\begin{aligned}
& w_{2 \kappa_{n}}(t) \geq \widetilde{M} \int_{\widetilde{J_{n}^{\prime}}} G^{*}(s, s) d s-\int_{\widehat{J_{n}^{\prime}}} G^{*}(s, s) a_{2}(s) d s \\
& +\widetilde{M} \int_{\widehat{J_{n}^{\prime}}} G^{*}(s, s) b_{21}(s) d s \\
& -\int_{\left(\widehat{\left.J_{n}^{\prime} \cap J_{n}\right)_{1}}\right.} G^{*}(s, s) b_{21}(s) d s \\
& \times\left\|w_{1 \kappa_{n}}(t)-\frac{b_{12}}{b_{11}} v_{n}\right\|_{\left(\widehat{\left.J_{n}^{\prime} \cap J_{n}\right)_{1}}\right.} \\
& -\int_{\left(\widehat{J_{n}^{\prime}}\right)_{1}} G^{*}(s, s) b_{22}(s) d s\left\|w_{2 \kappa_{n}}(t)+v_{n}\right\|_{\widehat{J_{n}^{\prime}}} \\
& -\int_{\widehat{J_{n}^{\prime} \backslash\left(\widehat{J_{n}^{\prime}}\right)_{1}}} G^{*}(s, s) b_{22}(s) d s \\
& \geq \widetilde{M} \int_{0}^{1} G^{*}(s, s) d s-\int_{0}^{1} G^{*}(s, s) a_{2}(s) d s \\
& +\widetilde{M} \int_{0}^{1} G^{*}(s, s) b_{21}(s) d s \\
& -\int_{0}^{1} G^{*}(s, s) b_{21}(s) d s \\
& \times\left(\left\|w_{1 \kappa_{n}}(t)\right\|_{{\widehat{J_{n}^{\prime}} \cap J_{n}}}+\left\|\frac{b_{12}}{b_{11}} v_{n}\right\|\right) \\
& -\int_{0}^{1} G^{*}(s, s) b_{22}(s) d s \\
& -\int_{0}^{1} G^{*}(s, s) b_{22}(s) d s\left(-\bar{m}_{2}\left(\kappa_{n}\right)-\left\|v_{n}\right\|\right) \\
& -\int_{0}^{1} G^{*}(s, s) b_{22}(s) d s \\
& =\widetilde{M}\left(\int_{0}^{1} G^{*}(s, s) d s+b_{21}\right)-a_{2}-b_{22} \\
& +\frac{\left\|v_{n}\right\|\left(b_{11} b_{22}-b_{12} b_{21}\right)}{b_{11}} \\
& -b_{21}\left\|w_{1 \kappa_{n}}(t)\right\|_{\widehat{J}_{n}^{\prime} \cap J_{n}}+b_{22} \bar{m}_{2}\left(\kappa_{n}\right) .
\end{aligned}
$$

Thus,

$$
\begin{aligned}
\bar{m}_{2}\left(\kappa_{n}\right)> & \widetilde{M}\left(\int_{0}^{1} G^{*}(s, s) d s+b_{21}\right)-a_{2}-b_{22} \\
& -b_{21}\left\|w_{1 \kappa_{n}}(t)\right\|_{\widehat{J}_{n}^{\prime} \cap J_{n}}+b_{22} \bar{m}_{2}\left(\kappa_{n}\right),
\end{aligned}
$$

which implies that

$$
\begin{aligned}
& \bar{m}_{2}\left(\kappa_{n}\right) \\
& >\frac{\widetilde{M}\left(\int_{0}^{1} G^{*}(s, s) d s+b_{21}\right)-a_{2}-b_{22}-b_{21}\left\|w_{1 \kappa_{n}}(t)\right\|_{\hat{J}_{n}^{\prime} \cap J_{n}}}{1-b_{22}} \\
& \text { If } \widehat{J_{n}^{\prime}} \cap J_{n}=\phi \text {, from }(85), \text { we have } \\
& \quad \bar{m}_{2}\left(\kappa_{n}\right)>\frac{\widetilde{M}\left(\int_{0}^{1} G^{*}(s, s) d s+b_{21}\right)-a_{2}-b_{22}}{1-b_{22}}
\end{aligned}
$$

which contradicts (79).

If $\widehat{J_{n}^{\prime}} \cap J_{n} \neq \phi$. Using a similar method of (85), we have

$$
\begin{aligned}
&-\left\|w_{1 \kappa_{n}}(t)\right\|_{\widehat{J}_{n}^{\prime} \cap J_{n}} \\
& \geq-\left\|w_{1 \kappa_{n}}(t)\right\|_{J_{n}} \\
&> \\
&>\frac{\widetilde{M}\left(\int_{0}^{1} G^{*}(s, s) d s+b_{12}\right)-a_{1}-b_{11}-b_{12} \bar{m}_{2}\left(\kappa_{n}\right)}{1-b_{11}} .
\end{aligned}
$$

Substituting it into (85), we obtain

$$
\begin{gathered}
\bar{m}_{2}\left(\kappa_{n}\right) \\
>\frac{\int_{0}^{1} G^{*}(s, s) d s\left(1-b_{11}-b_{21}\right)+b_{21}\left(1-b_{11}-b_{21}\right)}{\left(1-b_{22}\right)\left(1-b_{11}\right)-b_{21} b_{12}} \widetilde{M} \\
\quad-\frac{\left(a_{2}+b_{22}\right)\left(1-b_{11}\right)+\left(a_{1}+b_{11}\right) b_{21}}{\left(1-b_{22}\right)\left(1-b_{11}\right)-b_{21} b_{12}}
\end{gathered}
$$

which also contradicts (79). Therefore, $\lim _{v^{*} \rightarrow \infty} \vartheta=\infty$.

Now, we show that $\lim _{v^{*} \rightarrow \infty} \theta=-\infty$.

On the contrary, assume that there exists a vector sequence $\left\{\kappa_{n}\right\}=\left\{\left(\mu_{n}, v_{n}\right)\right\}$ such that $\mu_{n}=-\left(b_{12} / b_{11}\right) v_{n}$ and

$$
\lim _{v_{n} \rightarrow \infty} \theta_{n}=l>-\infty
$$

Similarly as before, it is impossible to have

$$
f_{1}\left(t, t^{\alpha-1}\left(W_{\kappa_{n}}(t)+\kappa_{n}\right)\right) \leq 0, \quad \forall t \in[0,1]
$$

as $\nu_{n}$ is sufficiently large.

Now for large $v_{n}$, we define

$$
\begin{aligned}
& \bar{I}_{n}=\left\{t \in[0,1]: f_{1}\left(t, t^{\alpha-1}\left(W_{\kappa_{n}}(t)+\kappa_{n}\right)\right)>0\right\}, \\
& \bar{I}_{n}^{\prime}=\left\{t \in[0,1]: f_{2}\left(t, t^{\alpha-1}\left(W_{\kappa_{n}}(t)+\kappa_{n}\right)\right)>0\right\} .
\end{aligned}
$$

Then, $\bar{I}_{n}$ is not empty. 
We can further divide the set $\bar{I}_{n}$ into two sets $\widetilde{\bar{I}}_{n}$ and $\widehat{\bar{I}}_{n}$ and divide the set $\bar{I}_{n}^{\prime}$ into two sets $\widetilde{\bar{I}}_{n}^{\prime}$ and $\hat{\bar{I}}_{n}^{\prime}$ as follows:

$$
\begin{aligned}
& \tilde{\bar{I}}_{n}=\left\{t \in \bar{I}_{n} \mid w_{1 \kappa_{n}}(t)-\frac{b_{12}}{b_{11}} v_{n} \leq 0\right\}, \\
& \hat{\bar{I}}_{n}=\left\{t \in I_{n} \mid w_{1 \kappa_{n}}(t)-\frac{b_{12}}{b_{11}} v_{n}>0\right\}, \\
& \tilde{\bar{I}}_{n}^{\prime}=\left\{t \in \bar{I}_{n}^{\prime} \mid w_{2 \kappa_{n}}(t)+v_{n} \leq 0\right\}, \\
& \tilde{\bar{I}}_{n}^{\prime}=\left\{t \in \bar{I}_{n}^{\prime} \mid w_{2 \kappa_{n}}(t)+v_{n}>0\right\} .
\end{aligned}
$$

It is easy to know that $\tilde{\bar{I}}_{n} \cap \hat{\bar{I}}_{n}=\phi, \tilde{\bar{I}}_{n}^{\prime} \cap \hat{\bar{I}}_{n}^{\prime}=\phi$ and $\bar{I}_{n}=\widetilde{\bar{I}}_{n} \cup \hat{\bar{I}}_{n}$, $\bar{I}_{n}^{\prime}=\tilde{\bar{I}}_{n}^{\prime} \cup \hat{\bar{I}}_{n}^{\prime}$.

Using a similar method of the proof of Lemma 12, we obtain that the set $\hat{\bar{I}}_{n}$ is not empty. Furthermore, there exists a constant $\widehat{M}>1$, independent of $t$ and $v_{n}$ such that

$$
\begin{aligned}
& f_{1}\left(t, t^{\alpha-1}\left(W_{\kappa_{n}}(t)+\kappa_{n}\right)\right) \leq \widehat{M}, \quad \text { for } t \in \widetilde{\bar{I}}_{n}, \\
& f_{2}\left(t, t^{\alpha-1}\left(W_{\kappa_{n}}(t)+\kappa_{n}\right)\right) \leq \widehat{M}, \quad \text { for } t \in \widetilde{\bar{I}}_{n}^{\prime}, \\
& w_{2 \kappa_{n}}(t)+v_{n} \leq \widehat{M}, \\
& \text { for } f_{2}\left(t, t^{\alpha-1}\left(W_{\kappa_{n}}(t)+\kappa_{n}\right)\right)<0, \quad t \in \widehat{\bar{I}}_{n}, \\
& w_{1 \kappa_{n}}(t)-\frac{b_{12}}{b_{11}} v_{n} \leq \widehat{M}, \\
& \text { for } f_{1}\left(t, t^{\alpha-1}\left(W_{\kappa_{n}}(t)+\kappa_{n}\right)\right)<0, \quad t \in \hat{\bar{I}}_{n}^{\prime} .
\end{aligned}
$$

Let

$$
\widehat{M}_{1}\left(\kappa_{n}\right)=\max _{t \in \bar{I}_{n}} w_{1 \kappa_{n}}(t) .
$$

From the definitions of $\tilde{\bar{I}}_{n}$ and $\hat{\bar{I}}_{n}$, we have

$$
\widehat{M}_{1}\left(\kappa_{n}\right)=\max _{t \in \overline{\bar{I}}_{n}} w_{1 \kappa_{n}}(t)=\left\|w_{1 \kappa_{n}}(t)\right\|_{\bar{I}_{n}} .
$$

Since $\widehat{\bar{I}}_{n}$ is not empty, it follows that $\widehat{M}_{1}\left(\kappa_{n}\right) \rightarrow \infty$ as $v_{n} \rightarrow$ $\infty$. Therefore, we can choose $v_{n 1}$ large enough so that

$$
\widehat{M}_{1}\left(\kappa_{n}\right)>\max \left\{1, \widehat{P}_{1}, \widehat{P}_{2}\right\},
$$

for $v_{n}>v_{n_{1}}$, where

$$
\begin{aligned}
\widehat{P}_{1}= & \frac{\widehat{M}\left(\int_{0}^{1} G^{*}(s, s) d s+b_{12}\right)+a_{1}+b_{11}}{1-b_{11}}, \\
\widehat{P}_{2}= & \frac{\int_{0}^{1} G^{*}(s, s) d s\left[\left(1-b_{22}\right)+b_{12}\right]+b_{12}\left(1-b_{22}+b_{21}\right)}{\left(1-b_{11}\right)\left(1-b_{22}\right)-b_{12} b_{21}} \widehat{M} \\
& +\frac{\left(a_{2}+b_{22}\right) b_{12}+\left(a_{1}+b_{11}\right)\left(1-b_{22}\right)}{\left(1-b_{11}\right)\left(1-b_{22}\right)-b_{12} b_{21}} .
\end{aligned}
$$

From $(H)$ and (42), we have

$$
\begin{aligned}
& w_{1 \kappa_{n}}(t) \\
& =\int_{0}^{1} G^{*}(t, s) f_{1}\left(s, s^{\alpha-1}\left(W_{\kappa_{n}}(s)+\kappa_{n}\right)\right) d s \\
& \leq \int_{\tilde{\bar{I}}_{n}} G^{*}(s, s) f_{1}\left(s, s^{\alpha-1}\left(W_{\kappa_{n}}(s)+\kappa_{n}\right)\right) d s \\
& \quad+\int_{\hat{\bar{I}}_{n}} G^{*}(s, s) a_{1}(s) d s \\
& \quad+\int_{\left.\hat{\bar{I}}_{n}\right)_{1}} G^{*}(s, s) b_{11}(s)\left|w_{1 \kappa_{n}}(s)-\frac{b_{12}}{b_{11}} v_{n}\right|^{p_{1}} d s \\
& \quad+\int_{\overline{\bar{I}}_{n} \backslash\left(\hat{\bar{I}}_{n}\right)_{1}} G^{*}(s, s) b_{11}(s)\left|w_{1 \kappa_{n}}(s)-\frac{b_{12}}{b_{11}} v_{n}\right|^{p_{1}} d s \\
& \quad+\int_{\left(\overline{\bar{I}}_{n} \cap \bar{I}_{n}^{\prime}\right)_{1}} G^{*}(s, s) b_{12}(s)\left|w_{2 \kappa_{n}}(s)+v_{n}\right|^{q_{1}} d s \\
& \quad+\int_{\left.\hat{\bar{I}}_{n} \cap\left([0,1] \backslash \bar{I}_{n}^{\prime}\right)\right) \cup\left(\left(\hat{\bar{I}}_{n} \cap \cap \bar{I}_{n}^{\prime}\right) \backslash\left(\hat{\bar{I}}_{n} \cap \bar{I}_{n}^{\prime}\right)_{1}\right)} G^{*}(s, s) b_{12}(s) \\
& \quad \times\left|w_{2 \kappa_{n}}(s)+v_{n}\right|^{q_{1}} d s,
\end{aligned}
$$

which follows from (93) and the definition in (63) that

$$
\begin{aligned}
w_{1 \kappa_{n}}(t) \leq & \int_{0}^{1} G^{*}(s, s) \widehat{M} d s+\int_{0}^{1} G^{*}(s, s) a_{1}(s) d s \\
& +\widehat{M} \int_{0}^{1} G^{*}(s, s) b_{12}(s) d s \\
& +\int_{\overline{\bar{I}}_{n}} G^{*}(s, s) b_{11}(s) d s\left(\widehat{m}_{1}\left(\kappa_{n}\right)-\left\|\frac{b_{12}}{b_{11}} v_{n}\right\|\right) \\
& +\int_{\overline{\bar{I}}_{n} \backslash\left(\hat{\bar{I}}_{n}\right)_{1}} G^{*}(s, s) b_{11}(s) d s \\
& +\int_{\left.\overline{\bar{I}}_{n} \cap \bar{I}_{n}^{\prime}\right)_{1}} G^{*}(s, s) b_{12}(s) d s \\
& \times\left(\left\|w_{2 \kappa_{n}}(t)\right\|_{\left(\overline{\bar{I}}_{n} \cap \bar{I}_{n}^{\prime}\right)_{1}}+\left\|v_{n}\right\|\right) .
\end{aligned}
$$

Thus,

$$
\begin{aligned}
w_{1 \kappa_{n}}(t) \leq & \int_{0}^{1} G^{*}(s, s) \widehat{M} d s+\int_{0}^{1} G^{*}(s, s) a_{1}(s) d s \\
& +\widehat{M} \int_{0}^{1} G^{*}(s, s) b_{12}(s) d s \\
& +\int_{0}^{1} G^{*}(s, s) b_{11}(s) d s\left(\widehat{m}_{1}\left(\kappa_{n}\right)-\left\|\frac{b_{12}}{b_{11}} v_{n}\right\|\right) \\
& +\int_{0}^{1} G^{*}(s, s) b_{11}(s) d s
\end{aligned}
$$




$$
\begin{aligned}
& +\int_{0}^{1} G^{*}(s, s) b_{12}(s) d s \\
& \times\left(\left\|w_{2 \kappa_{n}}(t)\right\|_{\left.\overline{\bar{I}}_{n} \cap \bar{I}_{n}^{\prime}\right)_{1}}+\left\|v_{n}\right\|\right) \\
& =\widehat{M}\left(\int_{0}^{1} G^{*}(s, s) d s+b_{12}\right)+a_{1}+b_{11} \\
& +b_{11} \widehat{M}_{1}\left(\kappa_{n}\right)+b_{12}\left\|w_{2 \kappa_{n}}(t)\right\|_{\overline{\bar{I}}_{n} \cap \bar{I}_{n}^{\prime}},
\end{aligned}
$$

which implies that

$$
\begin{aligned}
\widehat{M}_{1}\left(\kappa_{n}\right)< & \widehat{M}\left(\int_{0}^{1} G^{*}(s, s) d s+b_{12}\right)+a_{1}+b_{11}+b_{11} \widehat{M}_{1}\left(\kappa_{n}\right) \\
& +b_{12}\left\|w_{2 \kappa_{n}}(t)\right\|_{\overline{\bar{I}}_{n} \cap \bar{I}_{n}^{\prime}}
\end{aligned}
$$

Therefore, we have

$\widehat{M}_{1}\left(\kappa_{n}\right)$

$$
<\frac{\widehat{M}\left(\int_{0}^{1} G^{*}(s, s) d s+b_{12}\right)+a_{1}+b_{11}+b_{12}\left\|w_{2 \kappa_{n}}(t)\right\|_{\overline{\bar{I}}_{n} \cap \bar{I}_{n}^{\prime}}}{1-b_{11}} .
$$

If $\hat{\bar{I}}_{n} \cap \bar{I}_{n}^{\prime}=\phi$, then we have from (76) that

$$
\widehat{M}_{1}\left(\kappa_{n}\right)<\frac{\widehat{M}\left(\int_{0}^{1} G^{*}(s, s) d s+b_{12}\right)+a_{1}+b_{11}}{1-b_{11}},
$$

which contradicts (102).

If $\widehat{\bar{I}}_{n} \cap \bar{I}_{n}^{\prime} \neq \phi$. Using a similar method to that in Lemma 12, we have

$$
\begin{aligned}
& \widehat{M}_{1}\left(\kappa_{n}\right) \\
& <\frac{\int_{0}^{1} G^{*}(s, s) d s\left[\left(1-b_{22}\right)+b_{12}\right]+b_{12}\left(1-b_{22}+b_{21}\right)}{\left(1-b_{11}\right)\left(1-b_{22}\right)-b_{12} b_{21}} \widehat{M} \\
& \quad+\frac{\left(a_{2}+b_{22}\right) b_{12}+\left(a_{1}+b_{11}\right)\left(1-b_{22}\right)}{\left(1-b_{11}\right)\left(1-b_{22}\right)-b_{12} b_{21}}
\end{aligned}
$$

which also contradicts (96). Thus, the point $\widetilde{T}\left(-\mu^{*}, \nu^{*}\right)=$ $\left(-\left(b_{12} / b_{11}\right) v^{*}, v^{*}\right)$ lies in the second quadrant. The proof is completed.

Lemma 14. Suppose that $(H)$ and (9) hold. Then, for $v^{*}$ large enough, the line $P^{\prime} Q^{\prime}$ lies in the left of $\vartheta$-axis.

Proof. For any point $A(\theta, \vartheta)$ in $P^{\prime} Q^{\prime}$, it suffices to show that $\theta \rightarrow-\infty$ as $\nu^{*} \rightarrow \infty$ for any $\nu \in\left[-v^{*}, v^{*}\right]$.

On the contrary, we assume that there exists a vector sequence $\left\{\kappa_{n}\right\}=\left\{\left(\mu_{n}, v_{n}\right)\right\}$ satisfying $\mu_{n}=-\left(b_{12} / b_{11}\right) v_{n}$ and a point $v\left(v_{n}\right) \in\left[-v_{n}, v_{n}\right]$ such that $\theta\left(-\left(b_{12} / b_{11}\right) v_{n}, v\left(v_{n}\right)\right) \rightarrow$ $l>-\infty$ as $v_{n} \rightarrow \infty$. We define some sets $\bar{I}_{n}, \widetilde{\bar{I}}_{n}, \widehat{\bar{I}}_{n}$ and $\bar{I}_{n}^{\prime}$, $\widetilde{\bar{I}}_{n}^{\prime}, \hat{\bar{I}}_{n}^{\prime}$, and some numbers $\widehat{M}, \widehat{M}_{1}\left(\kappa_{n}\right)$ as in Lemma 13. Using a similar method of the proof of Lemma 13, we have

$$
\begin{aligned}
w_{1 \kappa_{n}}( & t) \\
\leq & \int_{\overline{\bar{I}}_{n}} G^{*}(s, s) f_{1}\left(s, s^{\alpha-1}\left(W_{\kappa_{n}}(s)+\kappa_{n}\right)\right) d s \\
& +\int_{\overline{\bar{I}}_{n}} G^{*}(s, s) a_{1}(s) d s \\
& +\int_{\left(\overline{\bar{I}}_{n}\right)_{1}} G^{*}(s, s) b_{11}(s)\left|w_{1 \kappa_{n}}(s)-\frac{b_{12}}{b_{11}} v_{n}\right|^{p_{1}} d s \\
& +\int_{\overline{\bar{I}}_{n} \backslash\left(\overline{\bar{I}}_{n}\right)_{1}} G^{*}(s, s) b_{11}(s)\left|w_{1 \kappa_{n}}(s)-\frac{b_{12}}{b_{11}} v_{n}\right|^{p_{1}} d s \\
& +\int_{\left(\overline{\bar{I}}_{n} \cap \bar{I}_{n}^{\prime}\right)_{1}} G^{*}(s, s) b_{12}(s)\left|w_{2 \kappa_{n}}(s)+v\left(v_{n}\right)\right|^{q_{1}} d s \\
& +\int_{\left.\hat{\bar{I}}_{n} \cap\left([0,1] \bar{I}_{n}^{\prime}\right)\right) \cup\left(\left(\hat{\bar{I}}_{n} \cap \bar{I}_{n}^{\prime}\right) \backslash\left(\overline{\bar{I}}_{n} \cap \bar{I}_{n}^{\prime}\right)_{1}\right)} G^{*}(s, s) b_{12}(s)
\end{aligned}
$$

It follows from (93)-(96) that

$$
\begin{aligned}
w_{1 \kappa_{n}}(t) \leq & \int_{0}^{1} G^{*}(s, s) \widehat{M} d s+\int_{0}^{1} G^{*}(s, s) a_{1}(s) d s \\
& +\widehat{M} \int_{0}^{1} G^{*}(s, s) b_{12}(s) d s \\
& +\int_{0}^{1} G^{*}(s, s) b_{11}(s) d s\left(\widehat{M}_{1}\left(\kappa_{n}\right)-\left\|\frac{b_{12}}{b_{11}} v_{n}\right\|\right) \\
& +\int_{0}^{1} G^{*}(s, s) b_{11}(s) d s \\
& +\int_{0}^{1} G^{*}(s, s) b_{12}(s) d s \\
& \times\left(\left\|w_{2 \kappa_{n}}(t)\right\|_{\overline{\bar{I}}_{n} \cap \bar{I}_{n}^{\prime}}+\left\|v\left(\nu_{n}\right)\right\|\right) .
\end{aligned}
$$

Notice that $-v_{n} \leq v\left(v_{n}\right) \leq v_{n}$; from (35), one gets

$$
\begin{aligned}
w_{1 \kappa_{n}}(t) \leq & \int_{0}^{1} G^{*}(s, s) \widehat{M} d s+a_{1}+\widehat{M} b_{12}+b_{11} \\
& +b_{11}\left(\widehat{M}_{1}\left(\kappa_{n}\right)-\left\|\frac{b_{12}}{b_{11}} v_{n}\right\|\right) \\
& +b_{12}\left(\left\|w_{2 \kappa_{n}}(t)\right\| \hat{\bar{I}}_{n} \cap \bar{I}_{n}^{\prime}+\left\|v_{n}\right\|\right) \\
= & \widehat{M}\left(\int_{0}^{1} G^{*}(s, s) d s+b_{12}\right)+a_{1}+b_{11} \\
& +b_{11} \widehat{M}_{1}\left(\kappa_{n}\right)+b_{12}\left\|w_{2 \kappa_{n}}(t)\right\| \hat{\bar{I}}_{n} \cap \bar{I}_{n}^{\prime},
\end{aligned}
$$


which implies that

$$
\begin{aligned}
& \widehat{M}_{1}\left(\kappa_{n}\right) \\
& \quad<\frac{\widehat{M}\left(\int_{0}^{1} G^{*}(s, s) d s+b_{12}\right)+a_{1}+b_{11}+b_{12}\left\|w_{2 \kappa_{n}}(t)\right\|_{\overline{\bar{I}}_{n} \cap \bar{I}_{n}^{\prime}}}{1-b_{11}} .
\end{aligned}
$$

It is easy to show that

$$
\widehat{M}_{1}\left(\kappa_{n}\right)<\frac{\widehat{M}\left(\int_{0}^{1} G^{*}(s, s) d s+b_{12}\right)+a_{1}+b_{11}}{1-b_{11}}
$$

for $\widehat{\bar{I}}_{n} \cap \bar{I}_{n}^{\prime}=\phi$, which contradicts (102), and

$$
\begin{aligned}
& \widehat{M}_{1}\left(\kappa_{n}\right) \\
& <\frac{\int_{0}^{1} G^{*}(s, s) d s\left[\left(1-b_{22}\right)+b_{12}\right]+b_{12}\left(1-b_{22}\right)+b_{12} b_{21}}{\left(1-b_{11}\right)\left(1-b_{22}\right)-b_{12} b_{21}} \widehat{M} \\
& \quad+\frac{\left(a_{2}+b_{22}\right) b_{12}+\left(a_{1}+b_{11}\right)\left(1-b_{22}\right)}{\left(1-b_{11}\right)\left(1-b_{22}\right)-b_{12} b_{21}}
\end{aligned}
$$

for $\hat{\bar{I}}_{n} \cap \bar{I}_{n}^{\prime} \neq \phi$, which contradicts (96) also. Thus, the line $P^{\prime} Q^{\prime}$ lies in the left of $\vartheta$-axis. The proof is completed.

Similar to the proof of Lemma 12, we can show that the image point $R^{\prime}$ of the point $R$ lies in the first quadrant. From (37), we have $\tilde{\mu}^{*}=\left(b_{22} / b_{21}\right) \nu^{*}$. Using a similar method of Lemma 13, we can show that the image point $S^{\prime}$ of the point $S$ lies in the fourth quadrant.

Using the conditions (36) and (37), similar to Lemma 14, we can show that the image line $Q^{\prime} R^{\prime}$ of the line $Q R$ lies above the $\theta$-axis, $R^{\prime} S^{\prime}$ lies in the right of the $\vartheta$-axis, and $S^{\prime} P^{\prime}$ lies under the $\theta$-axis. Therefore, we have the following lemmas.

Lemma 15. Suppose that $(H)$ and (9) hold. For $v^{*}$ large enough, $Q^{\prime} R^{\prime}$ lies above the $\theta$-axis, $Q^{\prime}$ lies in the second quadrant, and $R^{\prime}$ lies in the first quadrant.

Lemma 16. Suppose that $(H)$ and (9) hold. For $v^{*}$ large enough, $R^{\prime} S^{\prime}$ lies in the right of the 9 -axis and $S^{\prime}$ lies in the fourth quadrant.

Lemma 17. Suppose that $(H)$ and (9) hold. For $v^{*}$ large enough, $S^{\prime} P^{\prime}$ lies below the $\theta$-axis.

Proof of Theorem 1. From Lemmas 12-17, when $\mu^{*}, \nu^{*}$, and $\tilde{\mu}^{*}$ are large enough and satisfy (36) and (37), then the image $P^{\prime} Q^{\prime} R^{\prime} S^{\prime}$ of the curve $P Q R S$ will contain the zero in it. From Proposition 11, it follows that there exists a vector $\kappa_{0}=\left(\mu_{0}, \nu_{0}\right)$ such that the solution $W_{\kappa_{0}}(t)$ of (30) satisfies $W_{\kappa_{0}}(1)=0$, which implies that the integral equation (27) has a solution $Y(t)$. From (26), it follows that (19) has a solution. Therefore, the problem (1) has at least one solution. The proof is completed.

\section{Examples}

Example 1. Consider the following boundary value system:

$$
\begin{gathered}
D_{0+}^{3 / 2} x(t)+\frac{t^{1 / 3}}{2} x^{1 / 3}(t)+\frac{t^{1 / 3}}{4} \frac{y^{1 / 3}(t)}{1+\left|y^{1 / 3}(t)\right|}+t=0, \\
t \in(0,1), \\
D_{0+}^{3 / 2} y(t)+\frac{t^{1 / 3}}{3} \frac{x^{1 / 3}(t)}{1+\left|x^{1 / 3}(t)\right|}+\frac{t^{1 / 3}}{2} y^{1 / 3}(t)+\frac{t}{4}=0, \\
x(0)=y(0)=0, \quad x(1)=2^{1 / 2} x\left(\frac{1}{2}\right), \\
y(1)=2^{1 / 2} y\left(\frac{1}{2}\right),
\end{gathered}
$$

where

$$
\begin{aligned}
f_{1}\left(t, t^{\alpha-1}(x, y)\right) & =f_{1}\left(t, t^{1 / 2}(x, y)\right) \\
& =\frac{t^{1 / 2}}{2} x^{1 / 3}(t)+\frac{t^{1 / 2}}{4} \frac{y^{1 / 3}(t)}{1+t^{1 / 6}\left|y^{1 / 3}(t)\right|}+t \\
f_{2}\left(t, t^{\alpha-1}(x, y)\right) & =f_{2}\left(t, t^{1 / 2}(x, y)\right) \\
& =\frac{t^{1 / 2}}{3} \frac{x^{1 / 3}(t)}{1+t^{1 / 6}\left|x^{1 / 3}(t)\right|}+\frac{t^{1 / 2}}{2} y^{1 / 3}(t)+\frac{t}{4} .
\end{aligned}
$$

It is obvious that

$$
\begin{aligned}
& \left|f_{1}\left(t, t^{1 / 2}(x, y)\right)\right| \leq \frac{t^{1 / 2}}{2}|x(t)|^{1 / 3}+\frac{t^{1 / 2}}{4}|y(t)|^{1 / 3}+t \\
& \left|f_{2}\left(t, t^{1 / 2}(x, y)\right)\right| \leq \frac{t^{1 / 2}}{3}|x(t)|^{1 / 3}+\frac{t^{1 / 2}}{2}|y(t)|^{1 / 3}+\frac{t}{4}
\end{aligned}
$$

where

$$
\begin{gathered}
b_{11}(t)=\frac{t^{1 / 2}}{2}, \quad b_{12}(t)=\frac{t^{1 / 2}}{4} 4, \quad b_{21}(t)=\frac{t^{1 / 2}}{3}, \\
b_{22}(t)=\frac{t^{1 / 2}}{2}, \\
a_{1}(t)=t, \quad \eta=\frac{1}{2}, \\
p_{1}=p_{2}=q_{1}=q_{2}=\frac{1}{3} .
\end{gathered}
$$


It is easy to check that the function $f$ satisfies (8)-(12). Notice that

$$
\begin{aligned}
& G^{*}(s, s)= \frac{1}{\Gamma(1 / 2)\left(1-(1 / 2)^{1 / 2}\right)} \\
& \times \begin{cases}(1-s)^{1 / 2}-\left(\frac{1}{2}-s\right)^{1 / 2}, & 0 \leq s \leq \frac{1}{2} \\
(1-s)^{1 / 2}, & \frac{1}{2} \leq s \leq 1\end{cases} \\
& b_{21}(t), b_{12}(t)<b_{11}(t), b_{22}(t)=\max _{i, j=1,2}\left\{b_{i j}(t)\right\}=\frac{t^{1 / 2}}{2}
\end{aligned}
$$

Consider

$$
\begin{aligned}
\max _{1 \leq i \leq 2} & \int_{0}^{1} G^{*}(s, s)\left(b_{i 1}(s)+b_{i 2}(s)\right) d s \\
\leq & \int_{0}^{1} G^{*}(s, s)\left(\frac{s^{1 / 2}}{2}+\frac{s^{1 / 2}}{2}\right) d s \\
= & \int_{0}^{1} G^{*}(s, s) s^{1 / 2} d s \\
= & \frac{1}{\Gamma(1 / 2)\left(1-(1 / 2)^{1 / 2}\right)} \\
& \times\left(\int_{0}^{1}[(1-s) s]^{1 / 2} d s-\int_{0}^{1 / 2}\left[\left(\frac{1}{2}-s\right) s\right]^{1 / 2} d s\right) \\
= & \frac{1}{\sqrt{\pi}(1-\sqrt{2} / 2)}\left(\frac{\pi}{8}-\frac{\pi}{32}\right) \approx \frac{0.2945}{1.2533}<1
\end{aligned}
$$

which satisfies (9). Therefore, all conditions of Theorem 1 hold and thus the problem (111) has at least a solution.

\section{Conflict of Interests}

The authors declare that there is no conflict of interests regarding the publication of this paper.

\section{Acknowledgments}

The work was supported by the NSF of Hunan province (no. 13JJ3074), Major project of department of education of Hunan province (no. 13A088), the Scientific Research Foundation of Hengyang city (no. J1), and the Construct Program in USC.

\section{References}

[1] Y. Chen and X. Tang, "Solvability of sequential fractional order multi-point boundary value problems at resonance," Applied Mathematics and Computation, vol. 218, no. 14, pp. 7638-7648, 2012.

[2] Y. Chen and Z. Lv, "Solvability of fractional-order multipoint boundary-value problems at resonance on the half-line," Electronic Journal of Differential Equations, vol. 2012, no. 230, pp. 1-14, 2012.
[3] C. Huang, C. Peng, X. Chen, and F. Wen, "Dynamics analysis of a class of delayed economic model," Abstract and Applied Analysis, vol. 2013, Article ID 962738, 12 pages, 2013.

[4] C. Huang, X. Gong, X. Chen, and F. Wen, "Measuring and forecasting volatility in Chinese stock market using HAR-CJM model," Abstract and Applied Analysis, vol. 2013, Article ID 143194, 13 pages, 2013.

[5] C. Huang, Z. Yang, T. Yi, and X. Zou, "On the basins of attraction for a class of delay differential equations with nonmonotone bistable nonlinearities," Journal of Differential Equations, vol. 256, no. 7, pp. 2101-2114, 2014.

[6] F. Wen, Z. Li, C. Xie, and D. Shaw, "Study on the fractal and chaotic features of the Shanghai composite index," Fractals, vol. 20, no. 2, pp. 133-140, 2012.

[7] F. Wen and Z. Liu, "A copula-based correlation measure and its application in chinese stock market," International Journal of Information Technology \& Decision Making, vol. 8, no. 4, pp. 787-801, 2009.

[8] P. Amster, M. K. Kwong, and C. Rogers, "On a Neumann boundary value problem for the Painlevé II equation in twoion electro-diffusion," Nonlinear Analysis: Theory, Methods \& Applications, vol. 74, no. 9, pp. 2897-2907, 2011.

[9] C. Z. Bai, "Positive solutions for nonlinear fractional differential equations with coefficient that changes sign," Nonlinear Analysis: Theory, Methods \& Applications, vol. 64, no. 4, pp. 677-685, 2006.

[10] D. Delbosco, "Fractional calculus and function spaces," Journal of Fractional Calculus, vol. 6, pp. 45-53, 1994.

[11] A. A. Kilbas, H. M. Srivastava, and J. J. Trujillo, Theory and Applications of Fractional Differential Equations, vol. 204 of North-Holland Mathematics Studies, Elsevier, Amsterdam, The Netherlands, 2006.

[12] V. Lakshmikantham, S. Leela, and J. V. Devi, Theory of Fractional Dynamic Systems, Cambridge Scientiffic, 2009.

[13] V. Lakshmikantham and A. S. Vatsala, "Basic theory of fractional differential equations," Nonlinear Analysis: Theory, Methods \& Applications, vol. 69, no. 8, pp. 2677-2682, 2008.

[14] C. Z. Bai, "Existence of three solutions for a nonlinear fractional boundary value problem via a critical points theorem," Abstract and Applied Analysis, vol. 2012, Article ID 963105, 13 pages, 2012.

[15] V. Daftardar-Gejji, "Positive solutions of a system of nonautonomous fractional differential equations," Journal of Mathematical Analysis and Applications, vol. 302, no. 1, pp. 56-64, 2005.

[16] M. Belmekki and M. Benchohra, "Existence results for fractional order semilinear functional differential equations with nondense domain," Nonlinear Analysis: Theory, Methods \& Applications, vol. 72, no. 2, pp. 925-932, 2009.

[17] M. El-Shahed, "Positive solutions for boundary value problem of nonlinear fractional differential equation," Abstract and Applied Analysis, vol. 2007, Article ID 10368, 8 pages, 2007.

[18] V. D. Gejji, "Positive solutions of a system of non-autonomous fractional differential equations," Journal of Mathematical Analysis and Applications, vol. 302, no. 1, pp. 56-64, 2005.

[19] H. Jafari and V. D. Gejji, "Positive solutions of nonlinear fractional boundary value problems using Adomian decomposition method," Applied Mathematics and Computation, vol. 180, no. 2, pp. 700-706, 2006.

[20] E. R. Kaufmann and E. Mboumi, "Positive solutions of a boundary value problem for a nonlinear fractional differential equation," Electronic Journal of Qualitative Theory of Differential Equations, no. 3, pp. 1-11, 2008. 
[21] C. F. Li, X. N. Luo, and Y. Zhou, "Existence of positive solutions of the boundary value problem for nonlinear fractional differential equations," Computers \& Mathematics with Applications, vol. 59, no. 3, pp. 1363-1375, 2010.

[22] S. H. Liang and J. H. Zhang, "Positive solutions for boundary value problems of nonlinear fractional differential equation," Nonlinear Analysis: Theory, Methods \& Applications, vol. 71, no. 11, pp. 5545-5550, 2009.

[23] Z. G. Ouyang and C. H. Ou, "Existence of solutions for two nonlinear three-point boundary value problems with resonance," Communications in Applied Analysis, vol. 17, Article ID 47C60, 2013.

[24] S. Q. Zhang, "Positive solutions for boundary-value problems of nonlinear fractional differential equations," Electronic Journal of Differential Equations, vol. 2006, pp. 1-12, 2006.

[25] Y. Zhou and Y. Xu, "Positive solutions of three-point boundary value problems for systems of nonlinear second order ordinary differential equations," Journal of Mathematical Analysis and Applications, vol. 320, no. 2, pp. 578-590, 2006.

[26] Z. B. Bai, "On positive solutions of nonlocal fractional boundary value problem," Nonlinear Analysis: Theory, Methods \& Applications, vol. 72, no. 2, pp. 916-924, 2010.

[27] Z. B. Bai, "On solutions of some fractional $m$-point boundary value problems at resonance," Electronic Journal of Qualitative Theory of Differential Equations, no. 37, pp. 1-15, 2010.

[28] Y. H. Zhang and Z. B. Bai, "Existence of solutions for nonlinear fractional three-point boundary value problems at resonance," Journal of Applied Mathematics and Computing, vol. 36, no. 1-2, pp. 417-440, 2011.

[29] B. Ahmad and J. J. Nieto, "Existence results for a coupled system of nonlinear fractional differential equations with threepoint boundary conditions," Computers \& Mathematics with Applications, vol. 58, no. 9, pp. 1838-1843, 2009.

[30] J. J. Nieto and J. Pimentel, "Positive solutions of a fractional thermostat model," Boundary Value Problems, vol. 2013, article 5, 11 pages, 2013.

[31] J. J. Nieto, "Existence of a solution for a three-point boundary value problem for a second-order differential equation at resonance," Boundary Value Problems, vol. 2013, article 130, 7 pages, 2013.

[32] J. R. L. Webb and M. Zima, "Multiple positive solutions of resonant and non-resonant nonlocal boundary value problems," Nonlinear Analysis: Theory, Methods \& Applications, vol. 71, no. 3-4, pp. 1369-1378, 2009.

[33] R. P. Agarwal, M. Meehan, and D. O'Regan, Fixed Point Theorey and Applictions, Cambridge Unicersity Press, Cambridge, UK, 2001.

[34] Z. G. Ouyang and G. Z. Li, "Existence of the solutions for a class of nonlinear fractional order three-point boundary value problems with resonance," Boundary Value Problems, vol. 2012, article 68, 2012. 


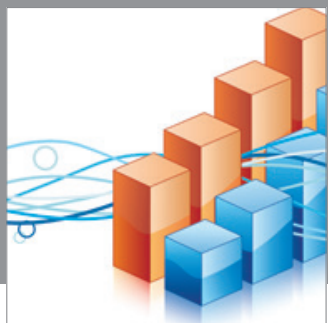

Advances in

Operations Research

mansans

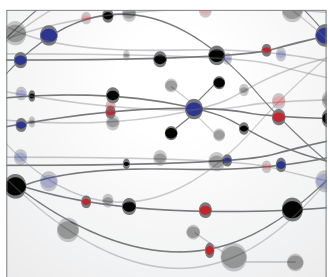

The Scientific World Journal
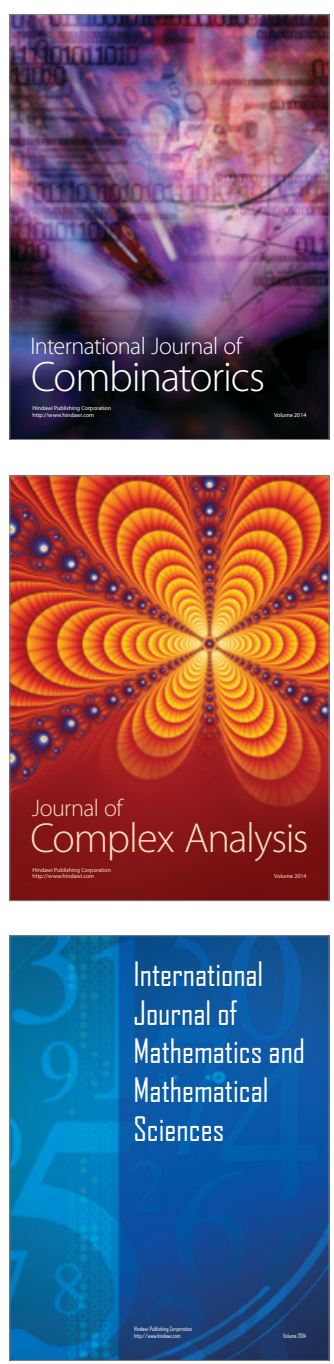
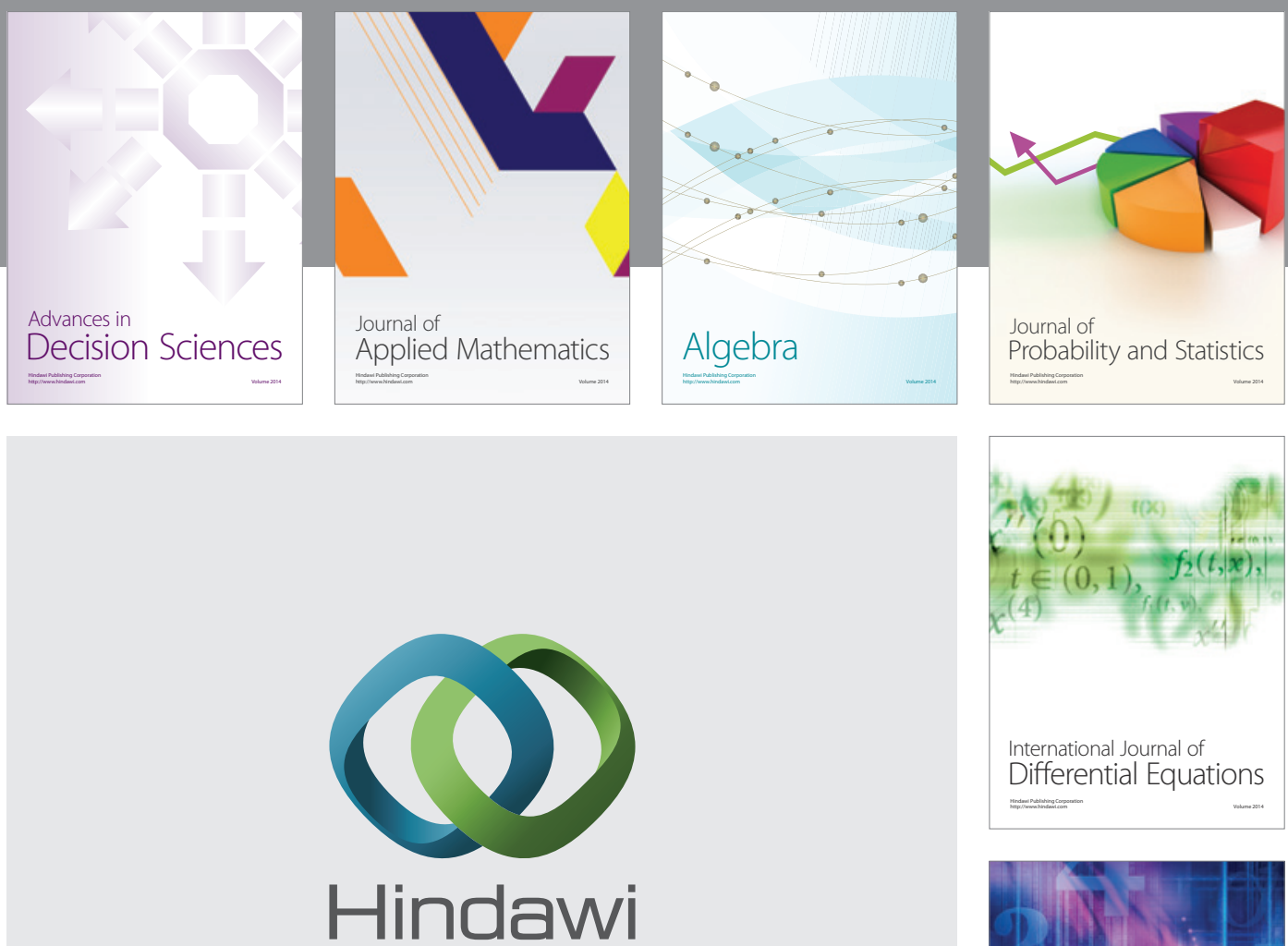

Submit your manuscripts at http://www.hindawi.com
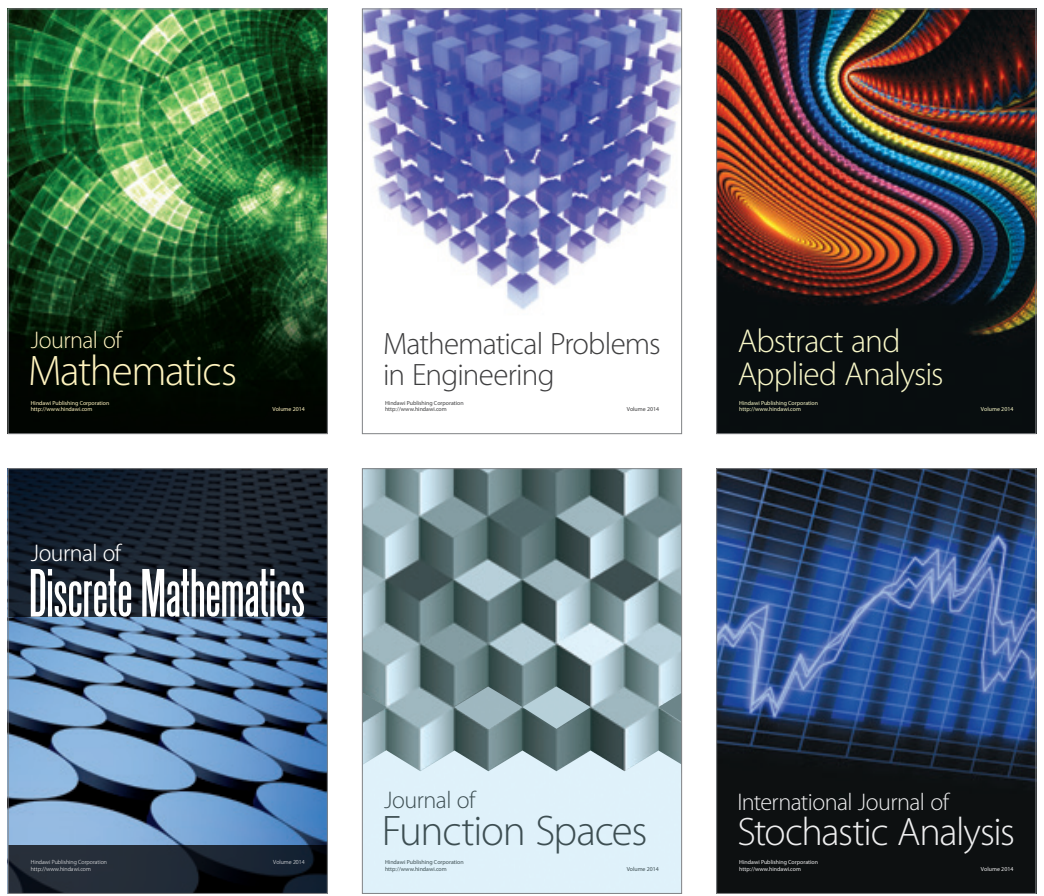

Journal of

Function Spaces

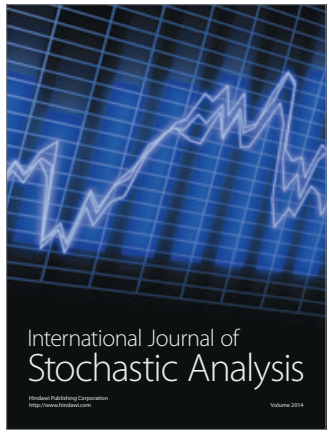

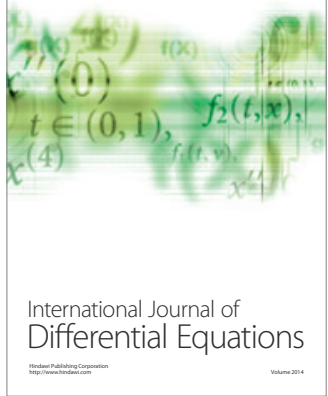
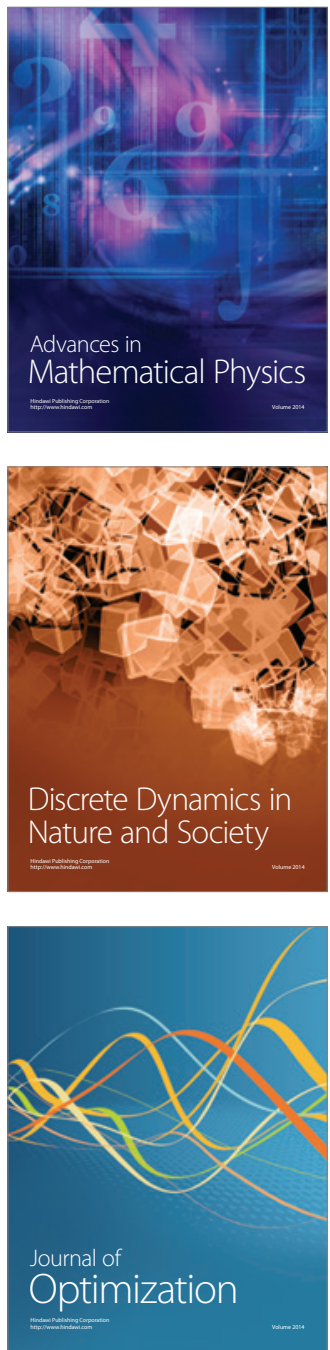